\title{
Interferometric observations of rapidly rotating stars
}

\author{
Gerard T. van Belle
}

Received: 10 October 2011 / Published online: 14 March 2012

(C) The Author(s) 2012. This article is published with open access at Springerlink.com

\begin{abstract}
Optical interferometry provides us with a unique opportunity to improve our understanding of stellar structure and evolution. Through direct observation of rotationally distorted photospheres at sub-milliarcsecond scales, we are now able to characterize latitude dependencies of stellar radius, temperature structure, and even energy transport. These detailed new views of stars are leading to revised thinking in a broad array of associated topics, such as spectroscopy, stellar evolution, and exoplanet detection. As newly advanced techniques and instrumentation mature, this topic in astronomy is poised to greatly expand in depth and influence.
\end{abstract}

Keywords Stars: rotation - Stars: imaging - Stars: fundamental parameters · Techniques: interferometric - Techniques: high angular resolution - Stars: individual: Altair, Alderamin, Achernar, Regulus, Vega, Rasalhague, Caph

\section{Introduction}

One of the most fundamental stellar characteristics that is most frequently taken for granted is the shape of a star. This is perhaps somewhat unsurprising - in our direct experience of seeing stellar disks, there is only the sun, which is very nearly a perfect sphere: on an average radius of $959^{\prime \prime} .28 \pm 0.15$ (Kuhn et al. 2004) there is only a variation of only $9.0 \pm 1.8$ mas (Rozelot et al. 2003) from equator to pole, indicating an oblateness $(b / a-1)$ of less than $10^{-5}$. However, for a surprisingly non-trivial number of stars, this degree of oblateness is in excess of $20 \%$ and, in certain cases,

Portions of this manuscript were prepared while in residence at European Southern Observatory Karl-Schwarzschild-Str. 2, 85748 Garching, Germany.

G.T. van Belle $(\bowtie)$

Lowell Observatory, 1400 West Mars Hill Road, Flagstaff, AZ 86001, USA

e-mail: gerard@lowell.edu 
even $30 \%$. Hints of this have been seen for decades in stellar spectroscopy, but this phenomenon has eluded direct observation until only recently.

The basis for departures from true sphericity lie in the rapid rotation of a star. Our sun turns about its axis at a stately rate of slightly more than once per month (Snodgrass and Ulrich 1990), which for a gaseous body of its size and mass leads to the $\sim 10^{-5}$ oblateness value seen above. However, as we shall see below, certain objects with greater mass are spinning at rates that are $10-60 \times$ more rapid, leading to distortions of their shapes.

Direct observation of these oblate spheroids has been a tantalizingly close possibility for decades, but it has only been within the last 10 years that there has been a sufficient convergence of theory, capability and technique for imaging at submilliarcsecond levels of resolution to be possible. The early spectroscopic results a century ago guided theoretical expectations, which then unfortunately had to lay dormant while the technology caught up. The advent of modern long-baseline interferometry in the visible and near-infrared has opened the door to directly characterize and even image these rapidly rotating stars_-and in particular, the most recent developments in this field of ultra-high-resolution astronomy have enabled rapid strides to be made in probing the surprising stellar structure of these objects.

In Sect. 2 we will explore the history of these developments, starting with the spectroscopic background (Sect. 2.1) which led to the early expectations and tests of interferometry (Sect. 2.2). The basic physics will be described in Sect. 3, including the simple Roche shape of a rigidly rotating gaseous spheroid (Sect. 3.1) and the resulting latitude dependence of flux known as the von Zeipel effect (Sect. 3.2). The specifics of interferometric observations of such targets is addressed in Sect. 4, including a general discussion of observational quantities (Sect. 4.1), and discussion of extensions of the technique to improved image reconstruction (Sect. 4.2) and greater spectral resolution (Sect. 4.3). A review of observational results to date is given in Sect. 5, followed by a discussion of the broad impact of these results to date Sect. 6; a summary of 191 future targets are suggested in Sect. 7.

\section{History}

\subsection{Spectroscopic underpinnings}

The earliest investigations into stellar rotation have their roots in Galileo's observations of sunspots (Drake 1957). ${ }^{1}$ The technique of spectroscopy was developed in the late 19th century, and was the necessary tool to quantitatively observe rotational effects on stars, rather than just our sun. However, prior to that development, there is clearly evidence for consideration of the effect of rotation in the intervening years upon other astronomical observables, such as photometry (cf. the work of Bouillaud

\footnotetext{
${ }^{1}$ There are, in fact, records of pre-'modern' sunspot observations taking place in ancient China. In the 4th century BC, astronomer Gan De from the State of Qi was the first to acknowledge sunspots as a solar phenomenon (Temple 1986); in the occident these were inaccurately viewed as obstructing natural satellites following the observations by the Benedictine monk Adelmus in 17-24 March 807 (Wilson 1917; Milone et al. 2008).
} 
in 1667 on Mira, and later by Cassini, Fontenelle, and Miraldi as presented in Brunet 1931). Thus, roughly a dozen generations after Galileo, Abney (1877) was the first to suggest axial rotation of stars could be observed from spectral line broadening. This suggestion was swiftly rebuked by Vogel (1877), who pointed out that broadened hydrogen lines suggested by Abney are frequently accompanied by other lines which themselves appear narrow-it was only later established that there are many stars in which all lines are broadened. An actual measurement of the rotation effect on spectral lines was carried out first by Schlesinger $(1909,1911)$ on the eclipsing binaries $\lambda$ Tauri and $\delta$ Libræ. In Schlesinger's observations, the less luminous companion occulted varying parts of the rapidly rotating primary, allowing measurement of variations in apparent radial velocity. This 'Rossiter-McLaughlin' effect (Rossiter 1924; McLaughlin 1924) is now a phenomenon commonly observed with transiting extrasolar planets, and is a useful tool for probing the alignment of the orbital plane relative to the stellar rotation axis (see, e.g., Winn et al. 2005).

The specific line shapes expected from rotational Doppler broadening were first predicted by Shajn and Struve (1929). Although their work emphasized once again the observation of this effect in binaries, they included relevant predictions for single stars. These line contours were used by Elvey (1930) in publishing the first list of rotational velocities (although there had been a few efforts on specific individual stars a few years before), and the rest of the decade saw rapid observational progress. Struve and Elvey (1931) linked rotation rate to spectral type, finding that A-type stars were the most likely fast rotators; Westgate (1933a, 1933b, 1934) published extensive observational catalogs for hundreds of stars between spectral types $\mathrm{O}$ and $\mathrm{F}$. These efforts solidified this new cornerstone of observational astronomy. Slettebak, in a dozen papers between 1949 and 1956, discovered that the most rapid rotators were to be found among Be stars, and established a relationship between rotation and mass.

Contemporaneously, von Zeipel (1924a, 1924b) demonstrated that (under the assumption of rigid body rotation) the local surface brightness at any point on a star is proportional to the local effective gravity, and as such, the temperature at the poles would be greater than at the equator for a rotating star. Slettebak (1949) went on to take the implications of this 'von Zeipel effect' and computed the first modifications for expected spectral line shapes of rapidly rotating, bright stars. These implications were developed in detail in Collins $(1963,1965)$ for continuum emission, and Collins and Harrington (1966), who incorporated shape distortion, aspect effects, gravity and limb darkening, and latitude variation in calculating $\mathrm{H}-\beta$ profiles. Harrington and Collins (1968) went on to characterize the intrinsic polarization expected for rapidly rotating early-type stars, and Collins and Sonneborn (1977) demonstrated that the spread of the main sequence (e.g. $M_{V}$ versus $b-y$ coordinates) due to rotation alone was 2 to 3 times larger than previously expected. This latter result had significant implications for inference of both ages and distance moduli for clusters of young stars.

Overall, the apparent position of stars on the HR diagram is significantly affected (e.g. 2-3 subtypes) in considering rapidly rotating stars to their nonrotating counterparts (see Slettebak et al. 1980; Collins and Smith 1985, and references therein). For the interested reader, the detailed history of line profile analysis is given a substantively more thorough treatment in Slettebak (1985). On 
the broad subject of rotation the multiple editions of Tassoul $(1978,2000)$ and proceedings of the two dedicated IAU meetings (Colloquium 4 in 1969 organized by Arne Slettebak, Symposium 215 in 2002 organized by Andre Maeder \& Philippe Eenens, both titled simply "Stellar Rotation") are indispensable resources.

\subsection{Interferometric observations}

It is unclear as to when the possibility of directly measuring stellar rotational distortion with optical interferometry ${ }^{2}$ was first considered as a plausible exercise. The pioneering angular diameter measurements of Michelson and Pease (1921) with the 20-foot beam interferometer on the Hooker 100" was the technology gate that opened up the possibility of such measurements. However, it is clear that the idea of potential observations did not fully develop until the guiding spectroscopic rotational velocities were themselves surveyed between 1930 and 1960, and the implications of the extremes of those velocities then evaluated. Furthermore, Pease's observational experience of operating the 50-foot beam interferometer, the successor to the 20-foot, was of extreme difficultly and highly limited success. Single measurements of individual stars were few in number, none were ever published in refereed journals (see the commentary in Townes 1999), and the collection of the sufficient data density necessary to establish a detection of rapid rotation on a given object was not forthcoming.

The entire endeavor of interferometric stellar angular diameter measurements lapsed into a state of dormancy for more than three decades, until the innovative proposal by Hanbury Brown and Twiss (1956) to pursue this task with intensity interferometry began to produce results. These efforts led to the construction and operation of the Narrabri Intensity Interferometer $\left(\mathrm{NI}^{2}\right)$, which produced the seminal results on single-star diameters (Hanbury Brown et al. 1974) and binary star orbits (HerbisonEvans et al. 1971). The success of $\mathrm{NI}^{2}$ in this regard, combined with the maturity of the underlying theory of rapid rotators that predicted observable effects, led to considerations of the possibility of using $\mathrm{NI}^{2}$ to observe the oblateness of Altair, as described in the PhD dissertations of Jordahl (1972) and Lake (1975). Unfortunately, the northern hemisphere location $\left(\delta=+8^{\circ} 52^{\prime}\right)$ of Altair is at odds with the southern location of Narrabri (latitude $=-30^{\circ} 19^{\prime}$ ), which limited $\mathrm{NI}^{2}$, s ability to collect a sufficient range of baseline projections upon the object. Simple diameter measurements from $\mathrm{NI}^{2}$ were published, but no detection of oblateness was made. (No mention of attempts to observe the more favorable declination, bright rapid rotator Acherner with $\mathrm{NI}^{2}$ is obvious in the literature.) Another contributing factor in this missed opportunity may have been the novel audacity of the intensity interferometer-and the degree to which its underlying physical principles were (and still are) poorly understood by the majority of other astronomers in the field. Gaining general acceptance of the simple single-star diameter measurements was difficult as it was, and a more

\footnotetext{
2 'Optical' interferometry is the term commonly used to refer to interferometry in the visible and nearinfrared. This technology family is separate from radio interferometry in its homodyne, rather than heterodyne, nature (e.g. mix-and-detect, rather than detect-and-mix).
} 
esoteric result like stellar oblateness was perhaps viewed as too ambitious. The completion of $\mathrm{NI}^{2}$ operations brought this second era of optical interferometry to a close with no detection of this phenomenon.

The third, and current, generation of optical interferometers has met with resounding success in probing more than just 1-dimensional parameterizations of stellar sizes. Many of the facilities in use today are characterized by multiple apertures, some even being relocatable, delivering dense data sets that permit sophisticated image reconstructions. An early attempt was made by Robert R. Thompson and myself using the IOTA interferometer in 1998 to detect the rotationally induced oblateness of S Cep, spending two weeks of observing using multiple baselines to characterize the shape of the object. Unfortunately, data on our check star SS Cep indicated spurious results, and the data had to be discarded.

Stellar oblateness was first detected when a team I led observed Altair with the Palomar Testbed Interferometer (PTI) on two separate baselines (van Belle et al. 2001), leading to disparate uniform-disk size measurements, particularly in comparison to the two contemporaneous size measurements on the check star Vega, which did agree. Extraction of a multi-parameter solution though use of Monte Carlo techniques, patterned after the similar approach applied to Keplerian orbit solving for interferometric data (Boden et al. 1999), indicated a $v \sin i=210 \pm 13 \mathrm{~km} \mathrm{~s}^{-1}$, in agreement with spectroscopic values. The data were insufficient to further constrain the additional parameters of inclination or gravity darkening, but a new sub-field in optical interferometry was opened up by that study.

Subsequent observations of rapidly rotating stars have been carried out with the more capable facilities VLTI, NOI, and (especially) the CHARA Array. These ensuing data sets have been sufficiently rich to allow for detailed parameterizations of the observed objects, including constraints on inclination and gravity darkening. A full discussion on the seven objects studied in-depth to date will be reviewed in Sect. 5, and is summarized in Table 1.

\section{Description of the geometry and basic physics}

\subsection{Roche model for stellar shape}

For a non-rotating star of mass $M$ in radiative equilibrium, its uniformly spherical shape of radius $R$ is trivially defined in terms of an equipotential surface (Eddington 1926):

$$
\Phi=\text { constant }=\frac{G M}{R}
$$

However, once rotation is imparted onto the star, a term must be added to account for the rotational potential:

$$
\Phi=\text { constant }=\frac{G M}{R(\theta)}+\frac{1}{2} \Omega^{2} R(\theta)^{2} \sin ^{2} \theta=\frac{G M}{R_{\text {pole }}}
$$




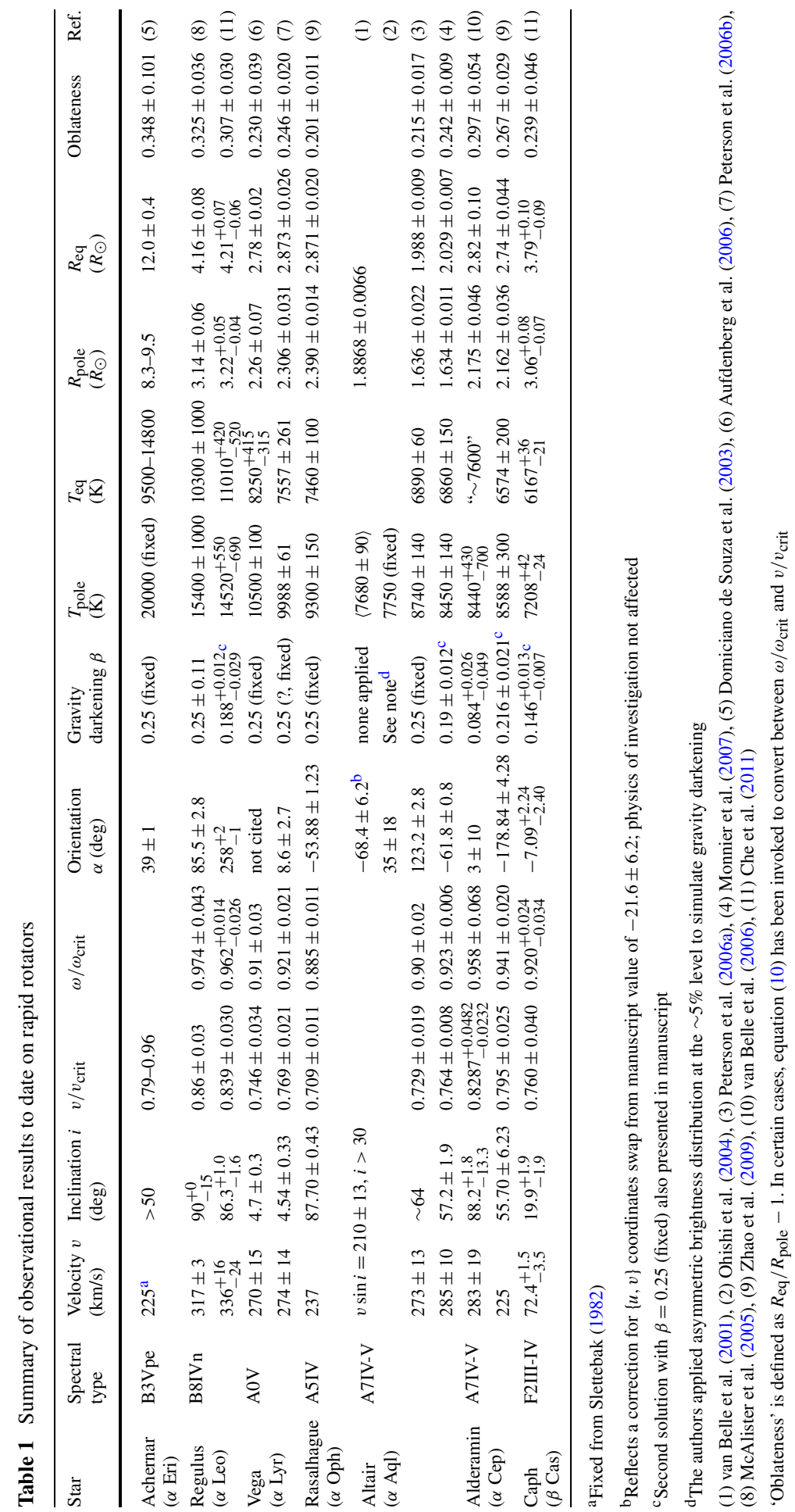




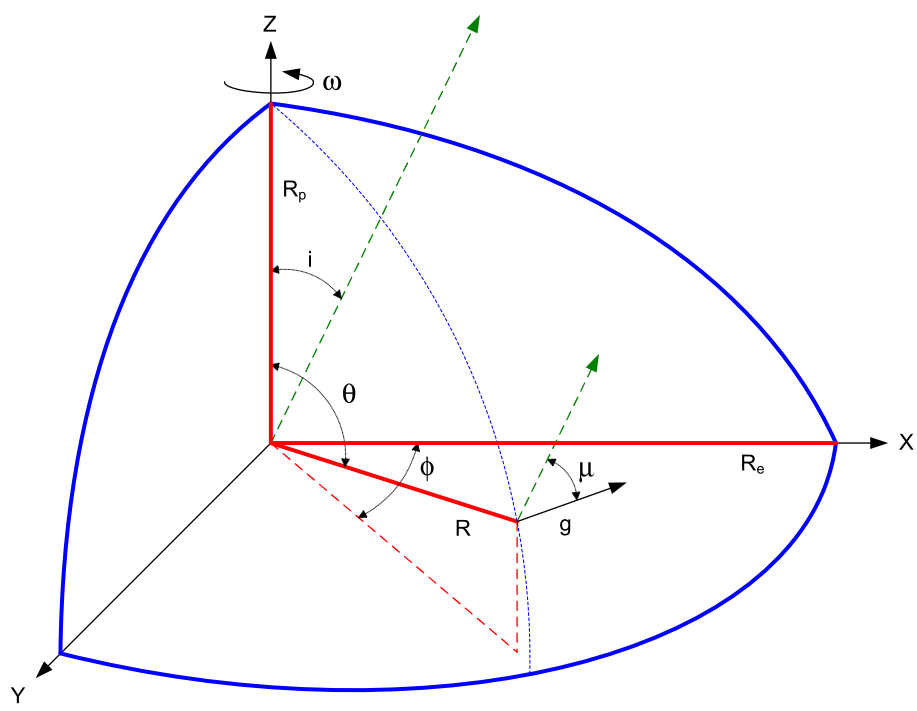

Fig. 1 Illustration of relevant rapid rotator geometry: observer is in XZ plane, with stellar polar axis of radius $R_{p}$ at inclination $i$ to observer. Stellar angular rotation rate $\omega$ (and stellar mass $M$ ) sets equatorial radius $R_{e}$. Stellar surface point at colatitude $\theta$, longitude $\phi$ has radius $R$ and local effective gravity vector $\vec{g}$, with $\mu$ being the cosine of the angle between $\vec{g}$ and the observer. Adopted to be consistent with Fig. 2 in Collins (1965)

where $\theta$ is the colatitude, $R_{\text {pole }}$ is the polar radius, $\Omega$ is the angular velocity (since our basic model here assumes uniform rotation, $\Omega \neq \Omega(\theta)$ ), and $R(\theta)$ is the radius at a given colatitude; this 'Roche model' (Roche 1837) geometry is illustrated in Fig. 1. The last equality in (2) comes from maintaining that equality across the stellar surface at the non-rotating pole. Another important caveat of this simple treatment is that polar radius will be treated as a constant, regardless of rotation speed. A more accurate examination of this overall phenomenon accounts for a decrease in polar radius as the rotating star approaches maximum speedthis effect is slight for most stars $(\lesssim 2 \%)$, although for $\lesssim 1 M_{\odot}$ objects this effect is thought to increase rapidly with decreasing mass to a maximum of $\sim 8 \%$ (Sackmann 1970; Deupree 2011). A important implication of advancing from (1) to (2) is that stellar models need to graduate from one-dimensional treatments to twodimensional.

The maximum "critical" angular velocity, $\Omega_{\text {crit }}$, where the outward centrifugal acceleration of rotation equals inward gravitational acceleration, can then be derived as

$$
\Omega_{\text {crit }}=\sqrt{\frac{8}{27} \frac{G M}{R_{\text {pole }}^{3}}}
$$


At this rotational speed the oblateness of the object is at its greatest, ${ }^{3}$ with $R_{\text {eq, critical }}=3 / 2 R_{\text {pole }}$. The fractional angular velocity $\omega$ is then defined as

$$
\omega \equiv \frac{\Omega}{\Omega_{\text {crit }}}
$$

and solving for the cubic equipotential $\Phi\left(R_{\text {pole }}\right)=\Phi(R, \theta)$ with trigonometric methods (see Collins 1963; Collins and Harrington 1966; Jordahl 1972), ${ }^{4}$ we arrive at an expression for the stellar radius at any colatitude $\theta$ for a given fractional rotational velocity $\omega$ :

$$
R(\omega, \theta)=\frac{3 R_{\text {pole }}}{\omega \sin \theta} \cos \left[\frac{\pi+\cos ^{-1}(\omega \sin \theta)}{3}\right]
$$

Owocki et al. (1994) identify the convenient identities

$$
\omega^{2}=\frac{27}{4} w_{0}\left(1-w_{0}\right)^{2}, \quad \frac{R_{\text {pole }}}{R_{\text {eq }}}=1-w_{0}
$$

where $w_{0} \equiv v_{\text {eq }}^{2} R_{\text {pole }} / 2 G M$ and $v_{\text {eq }}$ is the equatorial rotational velocity, presumably derived from spectroscopy; these identities allow use of the solution for $R(\omega, \theta)$ in (5). Figure 2 illustrates the expected distortion imparted upon a (Rocheapproximated) stellar surface as it progresses from a non-rotating to a rapidly rotating situation. For this object, an increase of rotational speed from $\omega=0$ to $\omega=0.92$ induces a $\sim 15 \%$ increase in the equatorial radius.

Owocki et al. (1994) point out that using the point-mass form for the gravitational potential found in (2) ignores high order multipole components that might arise from the rotationally distorted stellar mass distribution. However, Orlov (1961) shows that polytropic stellar structure models using the correct potential yield a change of less than $1 \%$ from the oblate surface radii predicted by (5).

To compute the surface gravity at a given colatitude, we compute the negative gradient of the effective potential in (2). The two components of the local effective gravity $\vec{g}$ at a given stellar colatitude $\theta$ in spherical polar coordinates are

$$
\begin{aligned}
& g_{r}(\theta)=-\frac{\delta \Phi}{\delta r}=-\frac{G M}{R(\theta)^{2}}+R(\theta)(\Omega \sin \theta)^{2} \\
& g_{\theta}(\theta)=-\frac{1}{r} \frac{\delta \Phi}{\delta \theta}=R(\theta) \Omega^{2} \sin \theta \cos \theta
\end{aligned}
$$

Thus $\vec{g}$, directed inwards along the local surface normal, is equal in magnitude to $\sqrt{g_{r}^{2}+g_{\theta}^{2}}$ (Cranmer and Owocki 1995; Aufdenberg et al. 2006).

The further details of the mathematics describing rotating Roche equipotential surfaces and it application to the stars can be found in Collins (1963), Collins and

\footnotetext{
${ }^{3}$ This choice of symbols is consistent with that found in the more recent works by Cranmer and Owocki (1995) and Aufdenberg et al. (2006), noting that the earlier manuscripts of Collins (1963, 1965); Jordahl (1972) differ slightly (e.g. ' $\omega$ ' is used in place of ' $\Omega$ ', ' $u$ ' is used instead of ' $\omega$ ', etc.)

${ }^{4}$ Aufdenberg et al. (2006) note a typographical error in (5) of Collins (1963); $\omega_{c}^{2}=G M / R_{e}^{3}$.
} 

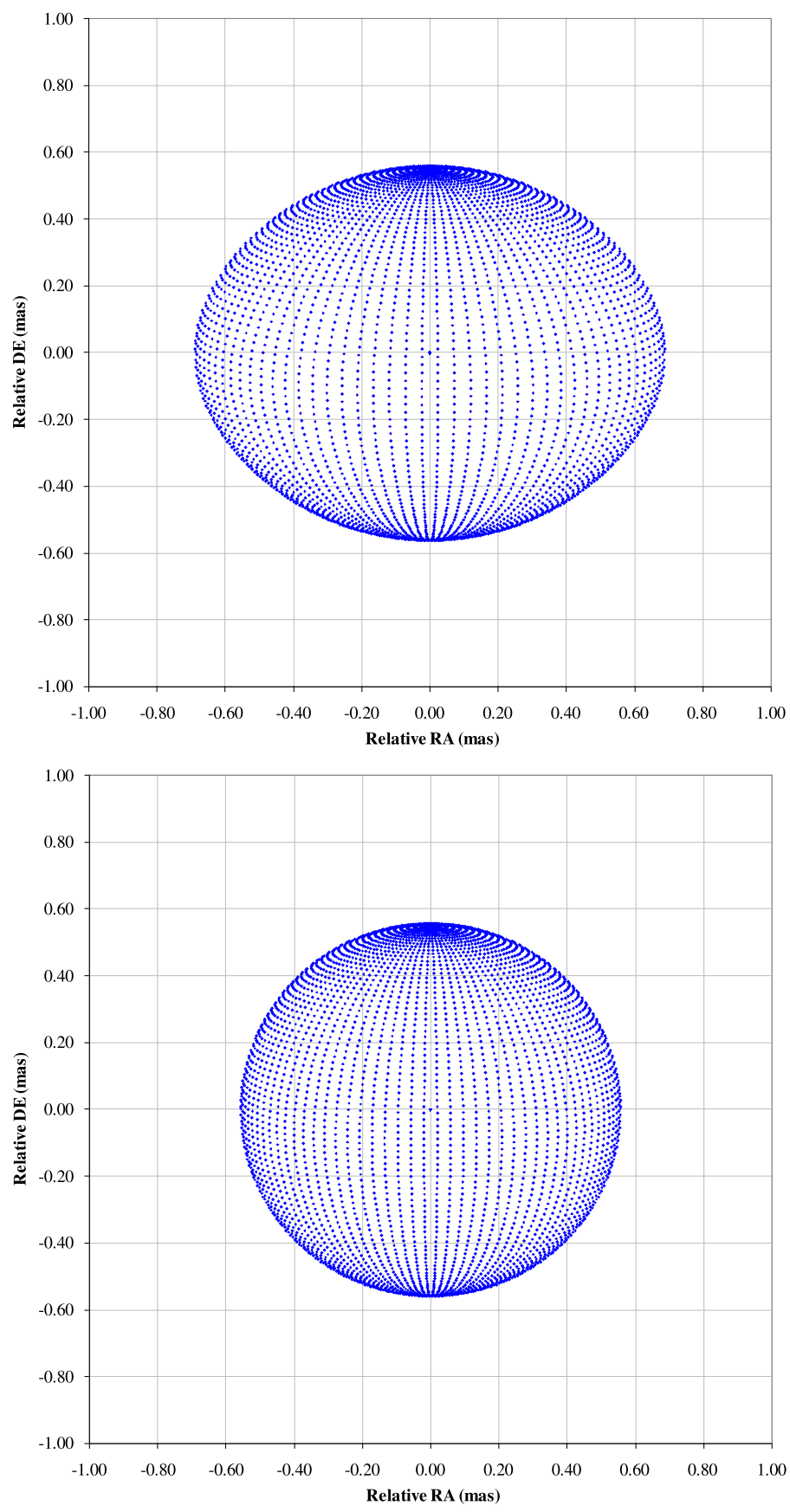

Fig. 2 Illustration of the phenomenon using a simple Roche model. Appearance is for two identical stars $\left(R_{\text {pole }}=3 R_{\odot}, d=25 \mathrm{pc}, \alpha=0^{\circ}, i=80^{\circ}\right.$ ); the star on the left is rotating with $\omega=0.92$, while the star on the right has only $\omega=0.001$. No contribution to pole flattening or consequences of limb and/or gravity darkening is included in this simple toy model 
Harrington (1966), Jordahl (1972), Tassoul (1978), Cranmer and Owocki (1995), van Belle et al. (2001), and Aufdenberg et al. (2006). Interesting related discussions regarding the oblateness of a planetary objects can also be found in the appropriate literature (e.g. Baron et al. 1989 wherein a discussion of Uranus' oblateness is considered).

For the benefit of the reader who often encounters rotational velocities from spectroscopy expressed in terms of $v \sin i$ (rather than angular velocity expressions), it is useful to note here a few relationships. Starting with (3), the critical linear velocity at the equator can be derived as

$$
v_{\text {crit }}=\sqrt{\frac{2}{3} \frac{G M}{R_{\text {pole }}}}=\sqrt{\frac{G M}{R_{\text {eq,critical }}}}
$$

Also, there is a subtle difference between fractional angular velocity and fractional linear velocity, since the linear radius that relates angular velocities to linear velocities is different between actual speed and the breakup speed:

$$
\frac{v}{v_{\text {crit }}}=\frac{\omega R_{\text {eq }}}{\omega_{\text {crit }} R_{\text {eq,crit }}}=\frac{\omega R_{\text {eq }}}{\omega_{\text {crit }} \frac{3}{2} R_{\text {pole }}}
$$

\subsection{The von Zeipel effect}

von Zeipel (1924a) and later Chandrasekhar (1933) showed that a rotationally oblate star has a local surface flux that is proportional to the local gravity, $F \propto g$. Thus, the Stefan-Boltzmann law, $F=\sigma_{\mathrm{SB}} T_{\text {eff }}^{4}$ implies that $T_{\text {eff }} \propto g^{0.25}$. This particular proportionality is applied in the case of radiative equilibrium; Lucy (1967) pointed out that for stars with convective envelopes, a weaker dependency is expected, with $T_{\text {eff }} \propto g^{0.08}$ given as 'representative' (although the value of 0.08 is weakly dependent upon mass, radius, luminosity, and other parameters). A closer examination of the Lucy (1967) discussion points out that progressing from slow to rapid stellar rotation should lead to pressure gradients that need to be balanced by the Coriolis force, with the consequence that the value of 0.08 quickly becoming less valid. A general parametrization expresses the relationship as $T_{\text {eff }} \propto g^{\beta}$, with $\beta$ either being a model predicted value or a parameter to fit as part of a larger overall solution for some set of observed astronomical data.

Thus, when considering the total flux from a rapidly rotating star, it is important to consider integrating over the surface accounting for the flux as it is tied to the local effective temperature. This colatitude-dependent temperature can be written as

$$
T_{\text {eff }}(\theta)=T_{\text {pole }}\left(\frac{g(\theta)}{g_{\text {pole }}}\right)^{\beta}
$$

allowing for a modification of the Stefan-Boltzmann law for characterization of the local stellar radiative flux,

$$
F(\theta)=\sigma_{\mathrm{SB}} T_{\mathrm{eff}}(\theta)^{4}
$$


The von Zeipel parameter $\beta$ has been tabulated for a range of stellar models varying in mass and age by Claret $(1998,2000) .{ }^{5}$ It is worth noting that the validity of (11) and (12) has been questioned by Connon Smith and Worley (1974) when the effects of differential rotation are considered.

For the case where the concerns regarding differential rotation may be neglected, Aufdenberg et al. (2006) use the preceding formalism to derive the temperature difference between the equator and pole:

$$
\Delta T_{\text {pole-eq }}=T_{\text {pole }}\left[1-\left(\frac{\omega^{2}}{\eta^{2}}-\frac{8}{27} \eta \omega\right)^{\beta}\right]
$$

where

$$
\eta=3 \cos \left[\frac{\pi+\cos ^{-1}(\omega)}{3}\right]
$$

It should be noted that use of a single ' $\beta$ ' term in describing a star perhaps oversimplifies the true nature of the stellar surface. As noted above, surfaces that are fully convective or fully radiative are expected to have values that are 0.08 and 0.25 , respectively. However, as the temperature ranges from hot to cool between a stellar pole and equator, the dominant mode of heat transportation may shift from radiative to convective, depending upon the value of those temperatures. One may easily conceive of an object whose temperature profile ranges pole to equator, straddling a 'sweet spot' value where such a shift occurs-the low latitudes would be convective, the high latitudes radiative. As such, a colatitude-dependent value of $\beta(\theta)$ may be appropriate to consider in constructing the most valid stellar model; the surface appearance of such an object will be most intriguing to image.

In light of the recent observational results that will be reviewed in Sect. 5, Espinosa Lara and Rieutord (2011) re-examine the underlying assumptions of the von Zeipel law that lead to the $T_{\mathrm{EFF}} \propto g_{\mathrm{EFF}}^{1 / 4}$ formulation. In particular, the 'strong hypothesis' of barotropicity (pressure only depends on the density) is already poor for slowly rotating stars (first noticed by Eddington 1925, see review in Rieutord 2006), and breaks down in noticeable ways for rapid rotators. These authors propose a new gravity darkening model, based on energy flux being a divergence-free vector antiparallel to the effective gravity, which appears to have good agreement with the interferometric results on Altair and Regulus.

\section{Interferometric observations of rapid rotators}

\subsection{Observational quantities, predictions}

Optical interferometers are, by nature, telescopes that can rightly be considered rather 'non-traditional'. Elements of an optical interferometer would be eminently familiar

\footnotetext{
${ }^{5}$ The gravity darkening parameter ' $\beta_{1}$ ' reported in Claret $(1998,2000)$ contains a factor of $4 \times$ relative to the $\beta$ used herein.
} 
to the non-specialist (e.g. the individual telescopes, for example), whereas there are also elements that would be rather alien (e.g. the beam-combining back end). This is also true of the elements of the data products-familiar concepts, such as photometry, quickly give way to unfamiliar ones, such as fringe visibility. For the purposes of this review, we will briefly introduce the relevant observational quantities in a concise manner, directing the reader to more thorough discussions should she or he be interested.

To begin on familiar territory, consider a 'normal' filled-aperture telescope. If it were to observe an unresolved point source, one would see on the telescope's image plane an Airy pattern with the characteristic width of $1.22 \lambda / D$ radians, where $\lambda$ is the wavelength of observation, and $D$ is the telescope aperture size. For a more complex object with on-sky brightness distribution $\mathrm{O}(\alpha, \delta)$ being observed by a telescope with point-spread function $\mathrm{P}(\alpha, \delta)$, the mathematical description of this process is

$$
\mathrm{I}(\alpha, \delta)=\iint \mathrm{P}\left(\alpha-\alpha^{\prime}, \delta-\delta^{\prime}\right) \mathrm{O}\left(\alpha^{\prime}, \delta^{\prime}\right) d \alpha^{\prime} d \delta^{\prime}
$$

For the simple case of a point source, $\mathrm{O}\left(\alpha^{\prime}, \delta^{\prime}\right)$ reduces to a Dirac delta function, $\mathrm{P}\left(\alpha-\alpha^{\prime}, \delta-\delta^{\prime}\right)$ is the point source function associated with a filled-aperture telescope, and $\mathrm{I}(\alpha, \delta)$ returns the Airy pattern.

The Fourier transform of (15) gives

$$
I(u, v)=T(u, v) \times O(u, v)
$$

which takes us directly to the points of interest for this review. Specifically, as a curiosity of their construction and measurement processes, optical interferometers tend to provide measurements that sample the Fourier information associated with an object, $I(u, v)$, rather than image plane information $(\mathrm{I}(\alpha, \delta))$. Secondly, the transfer function associated with an optical interferometer, $T(u, v)$, can be well-characterized. Thus, since the information associated with $I(u, v)$ and $T(u, v)$ can be collected, the nature of $O(u, v)$ can be computed — and by extension, the original object brightness distribution $\mathrm{O}(\alpha, \delta)$. For further expansive details on the underlying theory of optical interferometers, reference the van Cittert-Zernike theorem in a general optics textbook such as Born and Wolf (1980), or books specific to this topic (e.g. Goodman 2005). The proceedings of recent summer schools on optical interferometry are also very instructive in considering these instruments further (in particular, Lawson 2000; Haniff 2007a, 2007b), as is Bracewell (2000)'s book on the Fourier transform itself.

In practice, an optical interferometer takes light from two or more telescopes and recombines in such a way that the light from the apertures combines coherently, or interferes with itself (hence the name). Typically the interference criteria include maintaining wavefront quality, polarization, and path length from the point of light collection at the individual telescopes, through the system, to the point of beam recombination. In the simplest case of two telescopes, one may consider the interference of light as it is recombined and falls on a photodetector. Moving a mirror in the beam train that brought the light to the recombination point, the path length through the system may be swept through the point of equal path between the two arms of the interferometer, producing constructive and destructive interference. This characteristic pattern 
is referred to as a 'fringe', with its contrast (the intensity as measured by the photodetector between maximum constructive and maximum destructive interference) being the measured visibility:

$$
V=\frac{I_{\max }-I_{\min }}{I_{\max }+I_{\min }}
$$

As related to our discussion of (16), the visibility $V$ is the amplitude of a single point of complex image information $I(u, v)$. Fringe visibility is the basic observable of an optical interferometer and is directly related to the angular size of objects being observed by the instrument.

A second observable, closure phase, is also frequently measured by optical interferometers. Transmission of starlight through the turbulent atmosphere corrupts the phase information at optical wavelengths, so an absolute measurement of the phase information associated with $I(u, v)$ is not possible. However, with interferometers consisting of three or more telescopes, these corrupted phases can be summed about triangles to recover partial, uncorrupted phase information. If we consider a triangle of points from three telescopes $\{l, m, n\}$ that form baselines sampling the complex image $I(u, v)$, the measure phased for pair $(m, n)$ is $\psi_{m n}$, which consists of the source phase $\phi_{m n}$ and phase errors $\left(\xi_{m}-\xi_{n}\right)$. Thus, summing around a triangle of $\{u, v\}$ points,

$$
\begin{aligned}
C P_{l m n} & =\psi_{l m}+\psi_{m n}+\psi_{n l} \\
& =\phi_{l m}+\left(\xi_{l}-\xi_{m}\right)+\phi_{m n}+\left(\xi_{m}-\xi_{n}\right)+\phi_{n l}+\left(\xi_{n}-\xi_{l}\right) \\
& =\phi_{l m}+\phi_{m n}+\phi_{n l}
\end{aligned}
$$

we see that the measured sum $C P$ is directly related to the original uncorrupted $\phi$ values, since the $\xi$ errors cancel. Closure phase is an observable that is sensitive to the degree of asymmetry in an image $I(u, v)$. A good example of a striking closure phase signature is in the signal found in resolved stellar disk observations during planet transits, which can be considered 'perfect' star spots (van Belle 2008). Additionally, higher order such constructions, such as the closure amplitude $\left(C V_{l m n o}=V_{l m} V_{n o} / V_{l o} V_{m n}\right)$ are also possible but will not be considered herein.

To take this discussion of interferometric observables and place it in a context of actual measurements, we shall consider the representative, pioneering case of observing the rapid rotator Altair, which we shall see in Sect. 5 is a perennial favorite for demonstrating the prowess of an interferometer or instrument. In Fig. 3 we can see the basic appearance of Altair in the $\{u, v\}$ plane; the left panel is a representation of the visibility amplitude, and the right panel illustrates the visibility phase. These points were generated by construction of a Roche model based on the rotation parameters reported in Monnier et al. (2007) $\left(R_{\text {pole }}=1.634 R_{\odot}\right.$ and $\pi=194.45 \mathrm{mas}, \omega=0.923$, $i=57.2^{\circ}, \alpha=-61.8^{\circ}, \beta=0.19, T_{\text {pole }}=8450 \mathrm{~K}$ ), and using the prescription found in Sect. 4 of Aufdenberg et al. (2006) to generate colatitude dependent values for radius $R(\theta)$, local surface gravity $g(\theta)$, and local effective temperature $T_{\mathrm{EFF}}(\theta)$. (Limb darkening was ignored in this example, which has a small $\sim 1 \%$ effect on visibility amplitude measurements.) $T_{\mathrm{EFF}}$ was then converted to $1.6 \mu \mathrm{m}$ flux under the simplifying assumption of blackbody radiation (BBR). For a qualitative comparison of the 

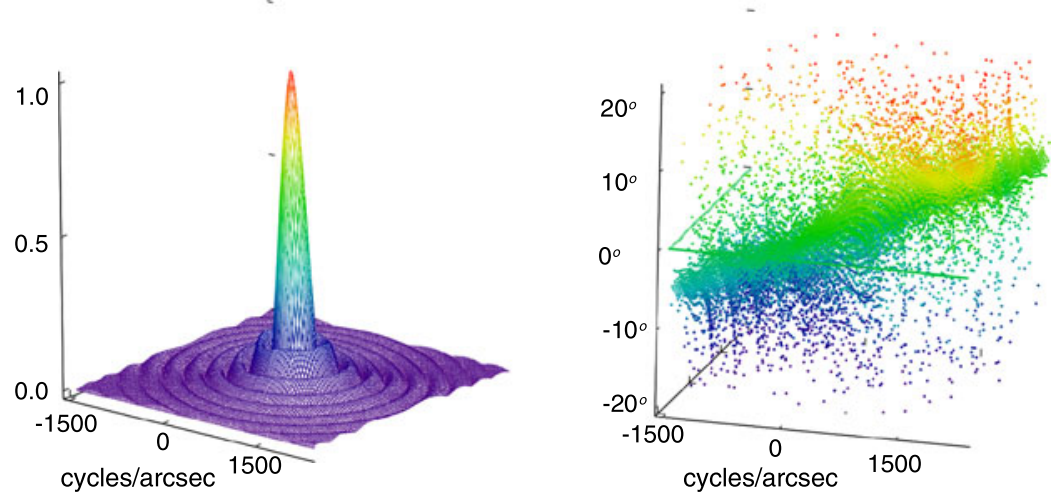

Fig. 3 The appearance of Altair in the $\{u, v\}$ plane, using parameters as reported by Monnier et al. (2007), with $R_{\text {pole }}=1.634 R_{\odot}$ (and $\pi=194.45$ mas), $\omega=0.923, i=57.2^{\circ}, \alpha=-61.8^{\circ}, \beta=0.19$, $T_{\text {pole }}=8450 \mathrm{~K}$; wavelength of observation is taken to be $\lambda=1.6 \mu \mathrm{m}$. The left panel is the modulus of the complex visibility (with visibility normalized to 1 at the center), and the right panel is the argument of the complex visibility (in units of degrees)
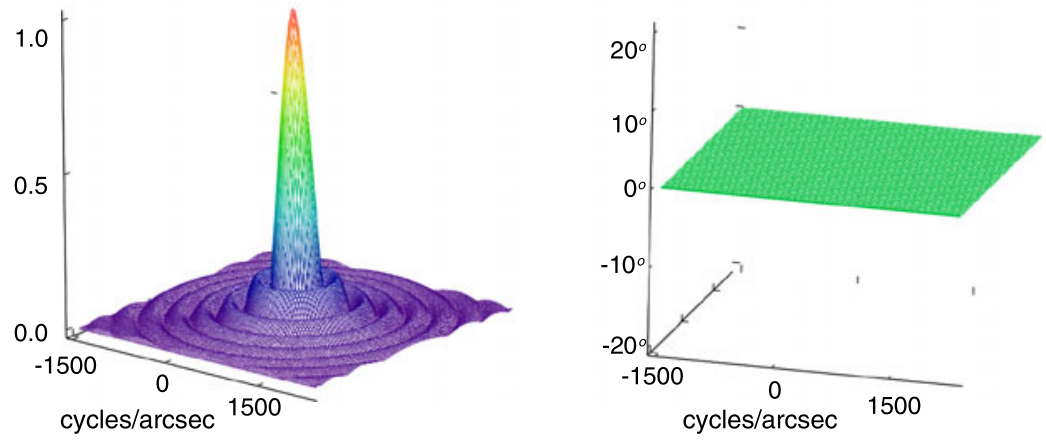

Fig. 4 As Fig. 3, but with $\omega=0.01$

effect rapid rotation has on these observables, a second nearly identical set of $\{u, v\}$ points was generated, with the exception that the rotation rate was artificially reduced from $\omega=0.923$ to $\omega=0.01$, in Fig. 4. The general deviation of these two figures are most clearly seen in the right-hand phase plots: the uniform source of Fig. 4 has a clear plane associated with a $\phi=0$ value, while the asymmetric appearance of the gravity-darkened rapid rotator in Fig. 3 shows a continuum of interesting phase values.

These differences, and the associated visibility amplitude excursions associated with the stellar shape produced by rapid rotation, are sharply highlighted in Fig. 5, which plots the difference of Figs. 3 and 4. Not only are the obvious phase excursions seen on the right, but previously hidden $\pm 10 \%$ visibility amplitude differences are made obvious. The deviations in visibility amplitude on the left a due primarily to the increased angular size on the sky in the stellar equatorial region; the closure phase 

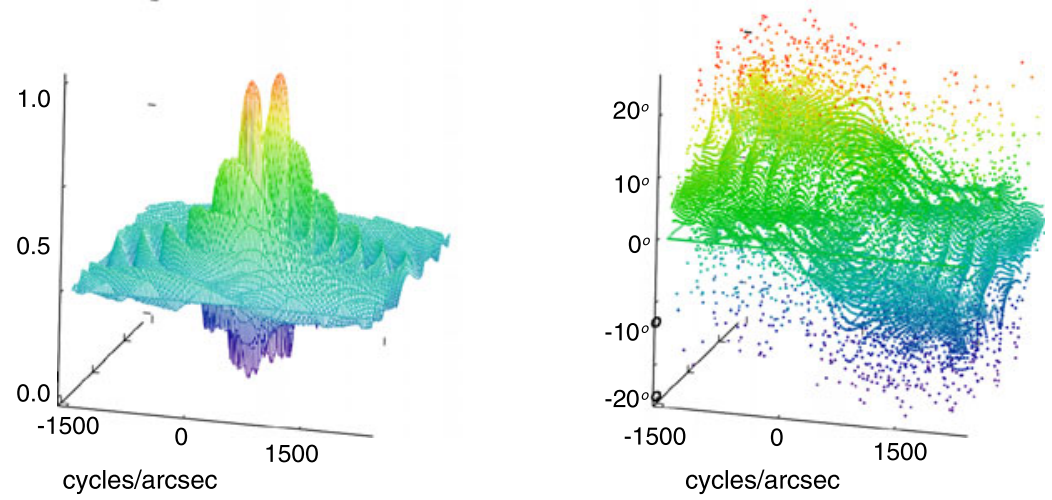

Fig. 5 The difference of the data sets seen in Figs. 3 and 4. The deviations in visibility amplitude on the left a due primarily to the increased angular size on the sky in the stellar equatorial region; the closure phase deviations on the right (in degrees) are most sensitive to the asymmetric brightness distribution due to the von Zeipel effect
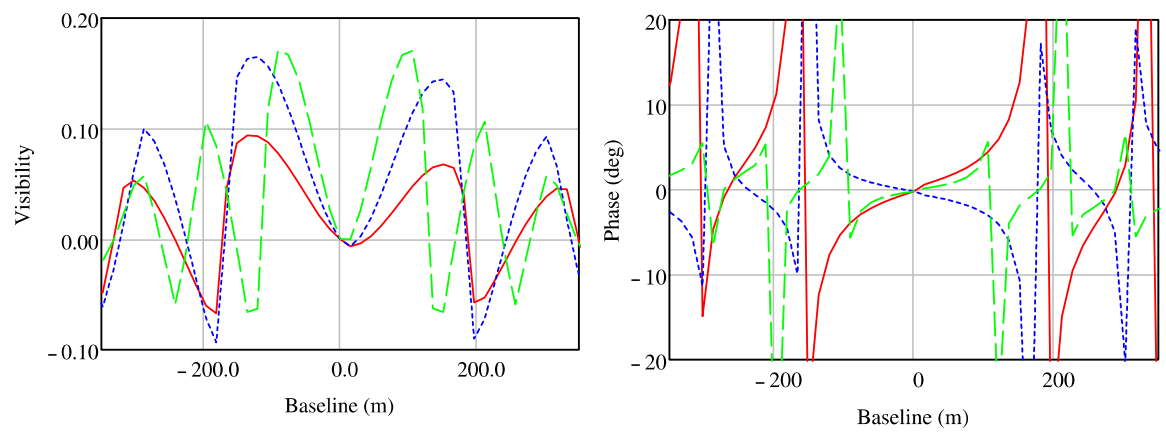

Fig. 6 Cuts along the $\{u, 0\},\{0, v\}$ and $\{u, v\}$ lines for the left and right panels of Fig. 5 (solid red, dotted blue, and dashed green, respectively). Abscissal units for both panes is in meters; the left-hand ordinate is normalized visibility, while the right-hand ordinate is in degrees

deviations on the right are most sensitive to the asymmetric brightness distribution due to the von Zeipel effect.

Finally, to connect the qualitative representations in these figures to some sense of what is detected by an optical interferometer, we plot in Fig. 6 cuts along the two planes of Fig. 5 along 3 lines $(\{u, 0\},\{0, v\}$ and $\{u, v\})$. We see that the observed visibility deviates for a rapid rotator from the values expected for a non-rotating object at the $\pm 10 \%$ level; the phase values vary by many tens, if not hundreds, of degrees - and as such, the resultant closure phase values would deviate on similar scales. Instruments such as CHARA Array MIRC and VLTI AMBER are able to measure visibility amplitude and closure phase; for the former, visibility amplitude precision is at the $\sim \sigma_{V}=5 \%$ level, and closure phase precision is as good as $\sim \sigma_{C P}=1^{\circ}$ for single-measurement accuracy with substantial $\sqrt{N}$ improvements possible (Zhao et al. 2011). Figures 7, 8, 9, 10, and 11 illustrate differences in visibil- 

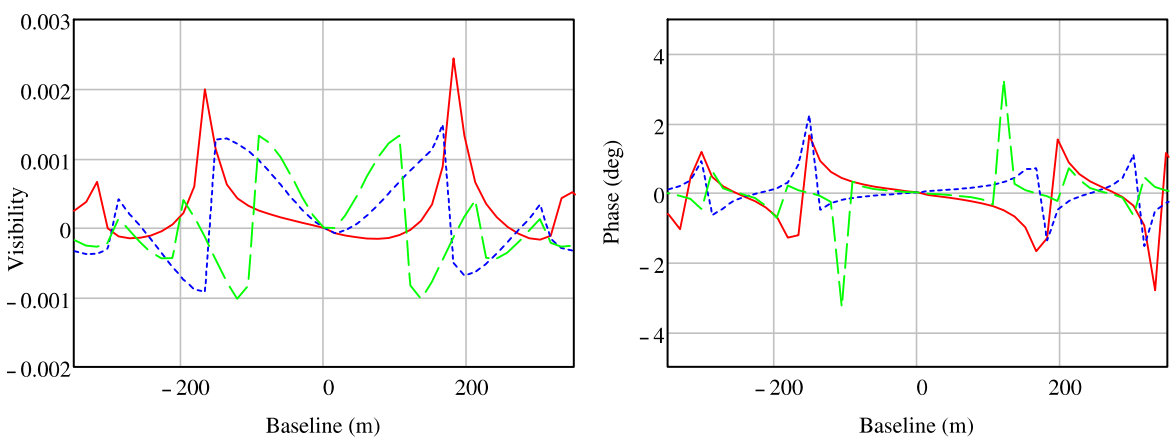

Fig. 7 Changes in visibility amplitude (left) and phase (right) for 1- $\sigma$ deviations in gravity darkening $\beta$. Lines are as in Fig. 6
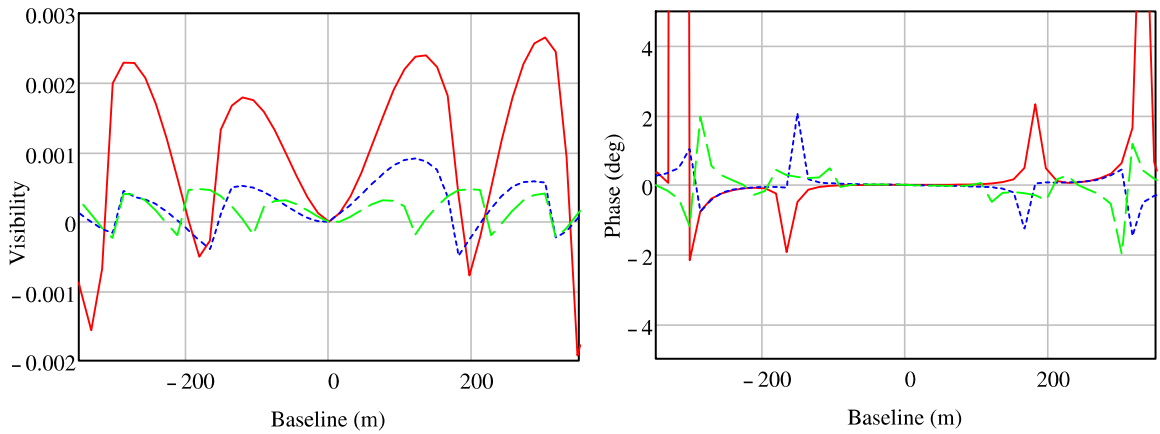

Fig. 8 Changes in visibility amplitude (left) and phase (right) for 1- $\sigma$ deviations in inclination $i$. Lines are as in Fig. 6
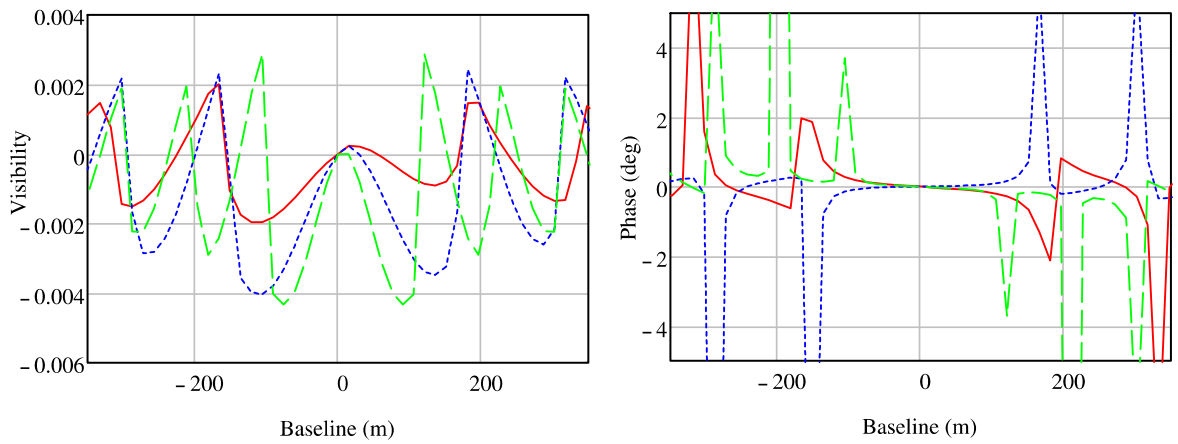

Fig. 9 Changes in visibility amplitude (left) and phase (right) for 1- $\sigma$ deviations in rotation rate $\omega$. Lines are as in Fig. 6

ity amplitude and phase (e.g. Fig. 3) as the solution parameters $\left\{\omega, R_{\text {pole }}, i, \alpha, \beta\right\}$ found in Monnier et al. (2007) are given 1- $\sigma$ excursions from their best-fit values. 

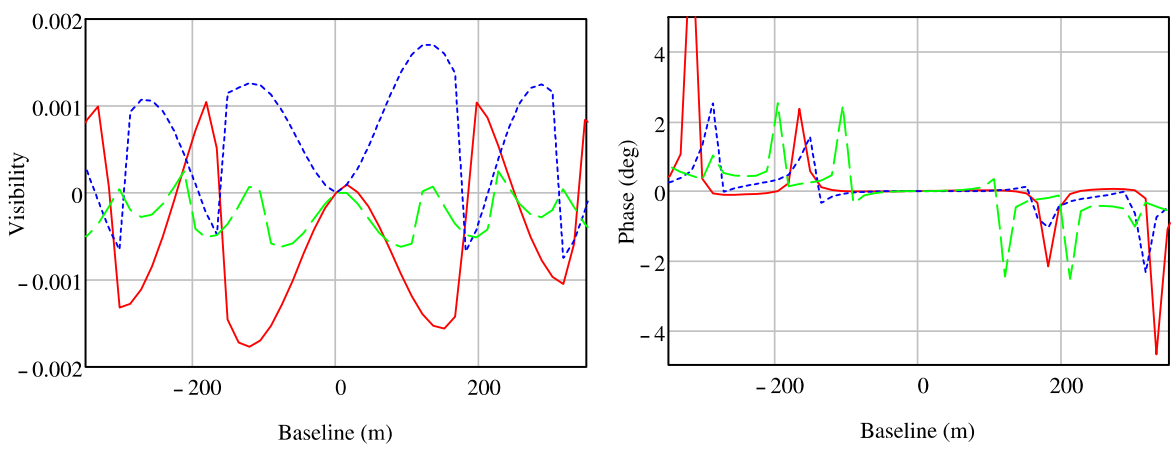

Fig. 10 Changes in visibility amplitude (left) and phase (right) for 1- $\sigma$ deviations in orientation $\alpha$. Lines are as in Fig. 6
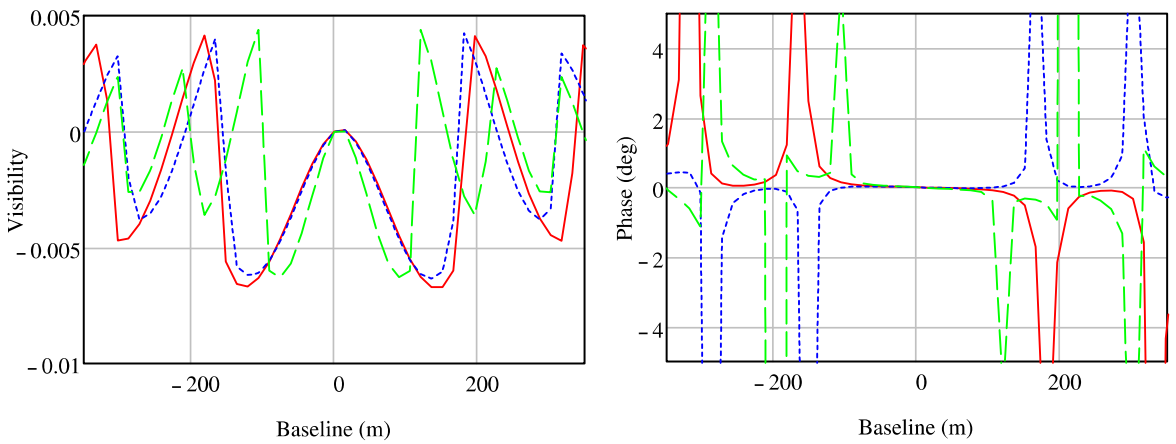

Fig. 11 Changes in visibility amplitude (left) and phase (right) for 1- $\sigma$ deviations in polar radius $R_{\text {pole }}$. Lines are as in Fig. 6

The practicalities of $\chi^{2}$ minimization of interferometric data are highlighted in Sect. 6 of van Belle et al. (2006) (henceforth referred to as VB06), which is illustrated pictorially in Fig. 12. VB06 used a Monte Carlo approach which began by constructing models of a rapidly rotating star based upon rotation $\omega$ and polar radius $R_{\text {pole }}$. Model surfaces were constructed for the star at some sufficiently dense interval (for VB06, the spacing was $0.8^{\circ}$ ) in both colatitude and longitude across the whole volume. Flux for a given surface area can then computed using the appropriate influence of gravity darkening as described in Sect. 3.2, with the $\beta$ parameter quantifying the effect, anchored to pole effective temperature $T_{\text {pole }}$. In addition to the gravity darkening, limb darkening appropriate for these model stars as indicated by the quadratic laws can be generated by Claret and Hauschildt (2003). These models are then mapped onto the sky, through the use of two additional free parameters describing inclination $i$ and on-sky rotational orientation $\alpha$.

Thus, for a given set of six randomized free parameters $\left\{\omega, R_{\text {pole }}, i, \alpha, \beta, T_{\text {pole }}\right\}$, a $\sim 100,000$ element volume surface is generated, projected upon the sky, rotated and the resultant image Fourier transformed for comparison to each of the observed visi- 


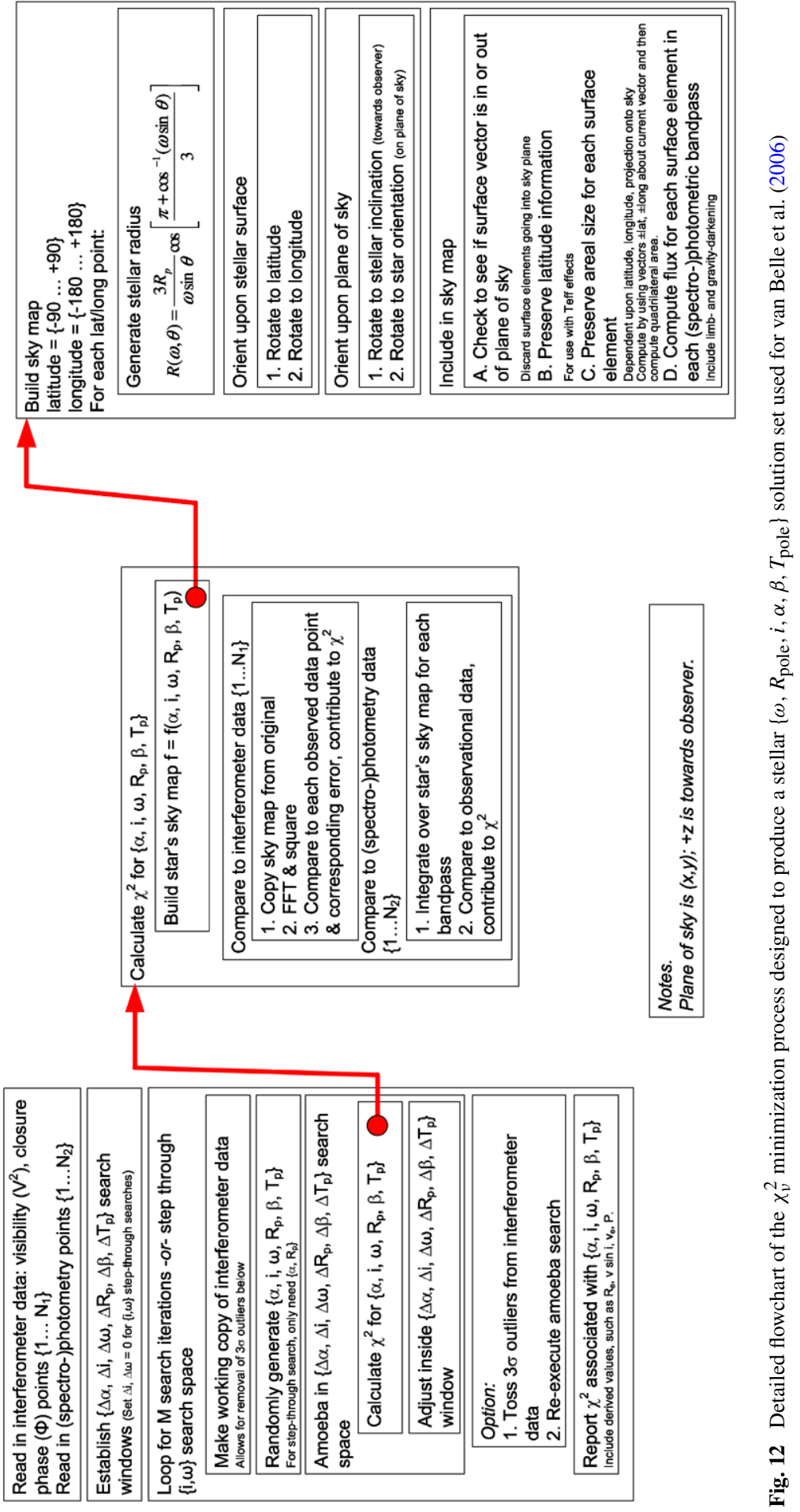


bility and closure phase data points, from which a $\chi^{2} / \mathrm{DOF}$ is calculated. As cleverly noted in Aufdenberg et al. (2006), the full Fourier transform of the model star's image does not need to be performed-only visibilities and closure phases associated with a small, specific set of $\{u, v\}$ points corresponding to the interferometer sampling need to be generated. The resulting computational load is substantially lighter and allows for faster and more complete Monte Carlo searches.

A detailed review of optical interferometry in astronomy can be found in Monnier (2003). A detailed discussion of phases in particular is continued in Monnier (2007), including expansive discussions on closure phase, and more esoteric observables possible with optical interferometers, such as differential closure phase, closure differential phase, and closure amplitudes.

\subsection{Image reconstruction}

Relative to the VB06 study, there are two specific extensions that can be made to this technique. First, inclusion of closure phase data can significantly expand the capability of the Monte Carlo routine to quickly recover the six characterizing parameters of the original star. The sensitivity of closure phase to asymmetries in brightness distribution speeds and improves the fidelity of the parametric fitting approach. The code written for VB06 in fact already includes generation of the closure phase data, but no comparison was made in that investigation since the CHARA Array's Classic pairwise beam combiner (from which the VB06 data were obtained) produces no measures of closure phase. However, both the CHARA Array's MIRC combiner and the VLTI AMBER instruments provide measurements of closure phase, and data from these instruments could be utilized for the parameter fitting; an example of this can be found in Sect. 4 of Zhao et al. (2009).

Second, and most instructively, optical interferometry instrumentation and techniques is advancing beyond simple pairwise telescope recombination to now include 3-, 4-, and even 6-way telescope recombination, which allows model-independent maps to be recovered from the data. These 'imaging' techniques are advancing rapidly (Cotton et al. 2008; Baron et al. 2010), and their great strength lies in the fact that they use little or no a priori assumptions, making them powerful, independent checks on the model-dependent approach to interpretation of interferometric data.

An excellent example of image reconstruction can be found in Sect. 3 of Zhao et al. (2009), who use the Maximum Entropy Method of radio synthesis imaging (Narayan and Nityananda 1986) as developed by Ireland et al. (2006) into the "Markov-Chain Imager for Optical Interferometry" (MACIM) code. Blind tests of the results from MACIM (Lawson et al. 2006) have shown it to be a robust tool for image reconstruction with optical interferometric data. For the application to rapid rotators, Zhao et al. (2009) incorporated an ellipse prior, justified by the reasonable expectation that the stellar photosphere has a sharp cutoff at the edge. This prior was generated through MACIM imaging of a grid of uniform surface brightness ellipses. Additionally, a Gaussian beam deweighting (as is common in radio synthesis imaging) was also applied, for image smoothness. Following these considerations, the final image 
was the one with the global maximum entropy. As closure phase precision progresses to levels much better than a fraction of a degree (Zhao et al. 2011), the fidelity of these image reconstructions will progress as well.

\subsection{Spectroscopy and spectro-interferometry}

Another ongoing, extremely powerful extension of these interferometric techniques is the combination of them with unresolved spectroscopy, and extension of the interferometric observables through spectral dispersion as well. For example, the rapidly rotating model generated to fit the interferometrically observed stellar surface brightness distribution of Vega (Aufdenberg et al. 2006) was upgraded to generate high dispersion spectra (Aufdenberg et al. 2007) and found that the high rotational velocitynecessary to reproduce the surface brightness distribution-was incompatible with the lower rotational velocity preferred by the line fits. These observations were taken to suggest that a simple von Zeipel gravity darkening law and uniform rotation are insufficient to describe the stellar photosphere. Yoon et al. (2010) demonstrate a further extension of the approach that attempts to unify high-resolution spectroscopy with interferometry, deducing that Vega's metallicity and mass are both lower than previously expected.

Additionally, the interferometric beam combiner may itself spectrally disperse the recombined light, permitting a wavelength-dependent analysis of the stellar surface brightness distribution. Sufficiently high-resolution dispersion, such as the $R \sim 12,000$ available with the VLTI AMBER instrument (Petrov et al. 2007), make it possible to examine spectral line profiles in a spatially resolved or even imaged sense. AMBER has already been used for spectro-interferometric observations of the Herbig Be star MWC 297 as this high spectral resolution (Weigelt et al. 2011).

\section{Stars studied to date}

An increasing number of facilities have been successfully employed to make a measurement of rotationally induced oblateness, starting in 2001 with the Palomar Testbed Interferometer (PTI, Colavita et al. 1999), the Very Large Telescope Interferometer (VLTI, Glindemann et al. 2003), the Navy Prototype Optical Interferometer (NOI, Armstrong et al. 1998), and by 2005 the Center for High Angular Resolution Astronomy (CHARA) Array (ten Brummelaar et al. 2005) was demonstrating the technique as well. These interferometric observations were interpreted with parametric fits similar to the example described in Sect. 4.1; recently, interferometric imaging (Sect. 4.2) has been demonstrated as a powerful tool in investigating these objects in a significantly more model-independent approach. Below we will review the studies to date; a timeline of these studies and their achievements is presented in Table 2.

\subsection{Altair}

Of the rapidly rotating stars, Altair ( $\alpha$ Aql, HR7557, HD187642; $\alpha=19^{\mathrm{h}} 50^{\mathrm{m}} 47.00^{\mathrm{s}}$, $\delta=+08^{\circ} 52^{\mathrm{m}} 05.96^{\mathrm{s}}$ ) is one of the nearest, brightest prototypes, and located close 
enough to the celestial equator to be observed by both northern- and southernhemisphere facilities $\left(d=5.143 \pm 0.024 \mathrm{pc}, m_{V}=0.77, m_{K}=0.10, \alpha=19^{\mathrm{h}} 50^{\prime}\right.$, $\delta=+08^{\circ} 52^{\prime}$; Perryman et al. 1997; Cutri et al. 2003). Altair is an A7IV-V star (Johnson and Morgan 1953), the 12th brightest of all stars, and has been known to be a rapid rotator for over half a century $\left(240 \mathrm{~km} \mathrm{~s}^{-1}\right.$ from Slettebak 1955). These characteristics made it an attractive target for the first interferometric observations of rapid rotation, and continue to make it appealing for instrument commissioning.

The interferometric observational implications were first considered theoretically in the PhD theses of Jordahl (1972) and Lake (1975), who investigated the effects that rapid rotation would have upon the shape, brightness distribution, and spectral features of that particular star. The observations of the object's size by the Narrabri Intensity Interferometer in 1965 were published (Hanbury Brown et al. 1974), but with only a uniform disk fit to the data of $2.78 \pm 0.13$ mas.

More than 25 years would pass between the publication of Hanbury Brown et al. (1974) and the first direct observation of rotational oblateness by van Belle et al. (2001), with Altair as the target. During that time, the possibility of making such a measurement was consciously known by investigators in the field, and attempts were even made at such a measurement on various targets, without success. However, using the Palomar Testbed Interferometer (PTI), we were able to take advantage of a number of aspects of the instrument to establish departures from spherical symmetry. First, PTI provided at the time of the observations two baselines of sufficient length $(>80 \mathrm{~m})$ to resolve the $\sim 3.2$ mas disk of Altair in the K-band. The baselines also provided sufficient $\{u, v\}$ coverage on the sky, such that the angle between those baselines when projected onto the sky was on order $\sim 50^{\circ}$. Second, the visibility data quality was such that the night-to-night repeatability was (under appropriate weather conditions) on order $\sigma_{V^{2}} \simeq 0.018$, which was necessary to compare the differing baseline's data with sufficient precision to indicate departures from spherical symmetry. PTI's highly automated operations also allowed for rapid collection of a large body of data points with each baseline as well. Finally, the nature of the experimental design showed the apparent change in interferometric visibility with sky projection angle was not seen with data collected contemporaneously on the check star, Vega, as expected from that star's small $v \sin i$ value $\left(21.9 \pm 0.1 \mathrm{~km} \mathrm{~s}^{-1}\right.$; Hill et al. 2004).

Collection of the observational data was only the first necessary step in achieving the confirmed detection of rotational oblateness. The proper direction for interpretation of the data was, at first, unclear, given the novel nature of the investigation at the time. Many of the publications of the time that discussed stellar rotation concentrated upon spectral line profile shapes but not the apparent shapes of the stellar disks. Indeed, some of the best early guidance was found in solar system literature discussing the shapes of the rapidly rotating gas giants (e.g., Baron et al. 1989). However, as recommended to us by George Collins (private communication), we found guidance in using the dissertation of Jordahl (1972) set against a computational methodology similar to that employed for reducing interferometric binary star data (e.g., Boden et al. 1999). This framework made it possible to extend the result from a mere toy model for a star with an elliptical on-sky appearance, to a physically meaningful family of dynamic Roche models of various inclinations with a single characteristic value of $v \sin i=210 \pm$ 
$13 \mathrm{~km} \mathrm{~s}^{-1}$, which was then compared, successfully, against the spectroscopic values.

This 2001 investigation contains one notable error, ${ }^{6}$ which fortunately does not alter the physics or the projected rotational velocity result: the $\{u, v\}$ coordinates were inadvertently swapped, resulting in an incorrect on-sky orientation angle (for example, Fig. 6 in that manuscript should be rotated about the $\alpha=\delta$ diagonal). The reported value in the manuscript of $-21.6 \pm 6.2 \mathrm{deg}$ corrects to $-68.4 \pm 6.2 \mathrm{deg}$ when this is taken into account.

This result on Altair quickly set the stage for further investigations of the phenomenon, with a variety of instruments and technique coming to bear on this particular object, along with other additional rapid rotators, beginning with Achernar the following year (as will be discussed in the next section, Sect. 5.2). This result was noted in the general astrophysical review literature as being a significant development (Trimble and Aschwanden 2003). With Altair, Ohishi et al. (2004) followed the initial result with observations of the star with the Navy Optical Interferometer (NOI). These investigators not only confirmed the oblate nature of the stellar photosphere, but reported a detection of a non-zero closure phase, consistent with a bright polar region produced by gravity darkening. This result was expanded upon with the addition of observations from the Very Large Telescope Interferometer (VLTI) by Domiciano de Souza et al. (2005), who synthesized together the PTI, NOI, and VLTI data sets into a unified solution. The following NOI observations and analysis by Peterson et al. (2006a) was able to unambiguously establish a stellar inclination for Altair.

Ultimately, these studies collectively set the stage for the ground-breaking study by Monnier et al. (2007), which was the first to directly image a main sequence star. The data from the MIRC instrument on the CHARA Array directly confirmed the presence of gravity darkening using an image reconstruction independent of the previous parametric modeling approaches, while at the same time providing evidence that departures from the standard formulation of that effect were insufficient to explain the observations. Using a value of $\beta=0.25$, the investigators were able to fit their data in a reasonably satisfactory way, but their goodness-of-fit criteria significantly improved when this was allowed to be a free parameter, which converged on a value of $\beta=0.190 \pm 0.012$. Differential rotation, opacity, and convection-related phenomena are all cited as possible reasons for this value of $\beta$; detailed spectrointerferometric line profile analysis (as noted in Sect. 4.3) is suggested as the preferred tool to disentangle which of these effects is the dominant one.

Given its size and brightness, one obvious further application of interferometric observations of Altair would be an attempt to directly observe stellar rotation with time-resolved imaging. The $\sim 10$ hour rotational period of star would have to be accounted for in such observations - no one exposure could be overly long, lest it risk smearing the image; 'snapshot' imaging would be a must. However, this could in fact be an advantage: during a single evening of observing, the full face of the star would be seen over time, allowing surface morphology maps to be built on a night-to-night

\footnotetext{
${ }^{6}$ Well, at least one-only one that we know about currently.
} 
basis. Such imaging would require a non-homogeneous surface, but that may be possible (even likely) given the known magnetic activity of Altair (Robrade and Schmitt 2009).

\subsection{Achernar ( $\alpha$ Eri)}

Following on the heels of the Altair observations, Domiciano de Souza et al. (2002) presented theoretical details on modeling rapid rotators which were soon applied to VLTI observations of the rapidly rotating Be star, Achernar ( $\alpha$ Eri, HR472, HD10144; $\alpha=01^{\mathrm{h}} 37^{\mathrm{m}} 42.85^{\mathrm{s}}, \delta=-57^{\circ} 14^{\mathrm{m}} 12.33^{\mathrm{s}}$; Domiciano de Souza et al. 2003). This object is the southern-hemisphere complement to the northern-hemisphere object Altair: it is bright and presents an remarkably large variation of angular size as a function of sky orientation. In fact, in evaluating their VLTI data as a solitary, rapidly rotating photosphere, the investigators found an extreme oblateness ratio of $a / b=1.56 \pm 0.05-a$ degree of photospheric distortion that could not be explained by Roche approximation. The authors claimed that the lack of $\mathrm{H} \gamma$ line emission during their observations was indicative of no disk contamination of the interferometric visibility data, and that "the classical assumption of the Roche approximation becomes questionable". Investigations of more exotic photospheric models were spurred onwards by this claim (e.g., Jackson et al. 2004).

However, ensuing investigations of this object (Kervella and Domiciano de Souza 2006; Carciofi et al. 2008) branched out to add contributions from a circumstellar environment (CSE) to model, which lessen the derived oblateness ratios; Kervella and Domiciano de Souza (2006) thereby derive the photospheric oblateness ratio dropped to $a / b=1.41 \pm 0.053$ with a photosphere-to-CSE relative flux ratio of $4.7 \pm 0.3 \%$ Additionally, there has been detection of a close-in companion by Kervella et al. (2008); however, inclusion of the companion and a rotationally distorted star alone in the visibility fits cannot explain the interferometry data, and a CSE contribution is still required (Kanaan et al. 2008). Companion thermal-IR interferometric observations with VLTI-MIDI has provided data consistent with extended emission associated with a fast wind ejected along the hot polar caps of star (Kervella et al. 2009).

Clearly this particular object has found a vigorous existence in the realm of interferometric study. However, in contrast to Altair, the complications of disks and winds have presented additional challenges (and rewards) for investigators attempting to fully fit models for Achernar. As the brightest Be star prototype, however, this object is uniquely situated to allow in-depth interferometric investigations to connect the effects of stellar rotation to the overall Be star phenomenon.

\subsection{Regulus $(\alpha$ Leo)}

As with Altair, Regulus ( $\alpha$ Leo, HR 3982, HD 87901, ADS 7654A; $\alpha=$ $\left.10^{\mathrm{h}} 08^{\mathrm{m}} 22.31^{\mathrm{s}}, \delta=+11^{\circ} 54^{\mathrm{m}} 01.95^{\mathrm{s}}\right)$ was observed by the Narrabri Intensity Interferometer (Hanbury Brown et al. 1974), with similar result: insufficient $\{u, v\}$ coverage prevented an in-depth characterization of this rapidly rotating star, although a gross size characterization was reported. Regulus had been recognized by Slettebak (1954) 


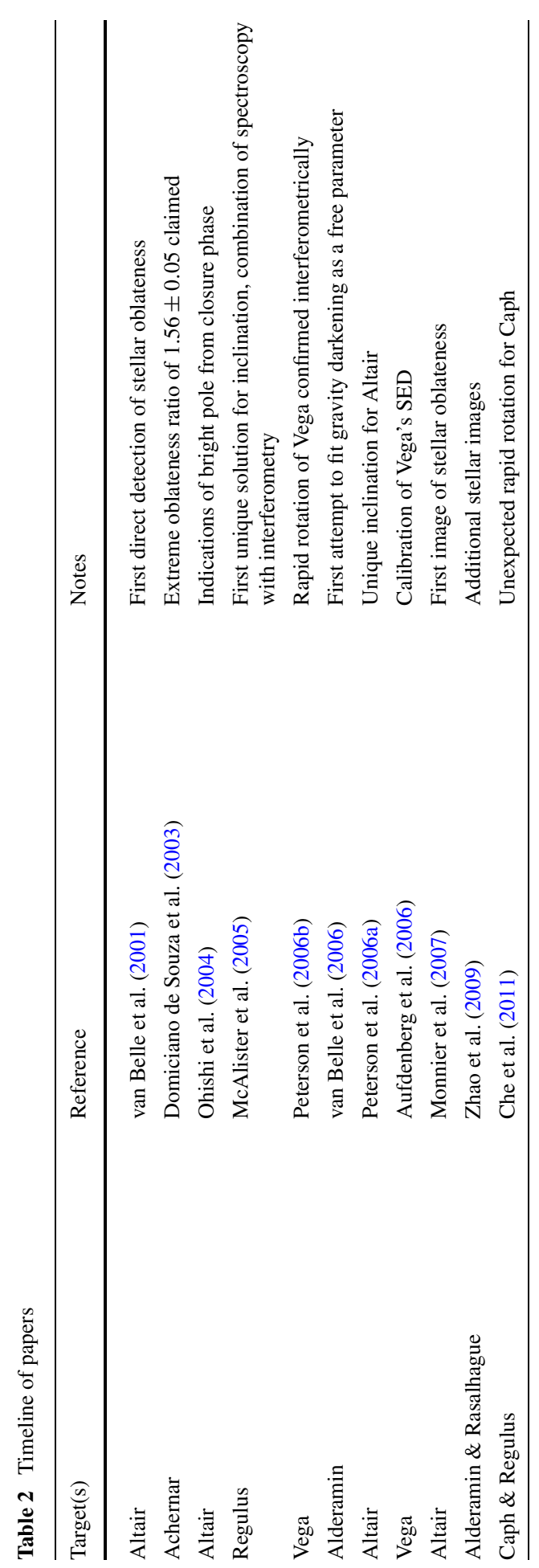


as a $\mathrm{B} 7 \mathrm{~V}$ rapid rotator with $v \sin i=350 \mathrm{~km} \mathrm{~s}^{-1}$, making this object an attractive target for such observing.

Observations of Regulus in 2004 were given the auspicious place of being the inaugural science investigation for the CHARA Array (McAlister et al. 2005), operated by the Georgia State University (ten Brummelaar et al. 2005). In addition to demonstrating the capabilities of the new array, this investigation was notable in the specific context of rapid rotator study for the following reasons: First, it marked the first time a combined interferometric and spectroscopic approach was applied to the data reduction methodology. Second, it was the first such investigation that resulted in a reasonably specific constraint on the inclination of the rotation axis for such an object. As of the publication of the Regulus results, previous investigations had painted broad suggestions on what various objects' true inclinations would be, but no specific values had be offered. The Regulus error was still quite generous $\left(i=90_{-15}^{+0} \mathrm{deg}\right)$ and yet specific, representing one of the ultimate benefits of this manner of investigation.

The resulting solution set for Regulus is a rather rich characterization of the object, including values for inclination $(i)$, on-sky orientation $(\alpha)$, gravity darkening $(\beta)$, rotational velocity $(v)$, fractional rotational velocity $\left(v_{e} / v_{c}\right)$, polar and equatorial radii $\left(R_{p}, R_{e}\right)$, polar and equatorial temperatures $\left(T_{p}, T_{e}\right)$, mass $(M)$, luminosity $(L)$, and interstellar extinction $A_{V}$. Such a rich data set allows for in-depth evaluation of the object on a variety of fronts. For example, comparison of the on-sky orientation with its known proper motion shows that Regulus's space motion is roughly along the axis of its rotational spin. This observation is thought-provoking from the context of considering the formation history of the star and the dynamics of its protostellar cloud.

More specifically, providing a the true inclination solution had far-reaching consequences. From the inclination, the true rotation velocity and fractional rotational velocity could be established. The degree to which such objects are rotating close to their breakup velocity has significant implications regarding the nature of mass loss in Be stars (e.g., Porter and Rivinius 2003). The range in temperature from the pole to the equator has cast doubt on the 'young' age estimate of $150 \mathrm{Myr}$ (Gerbaldi et al. 2001) and a significantly older estimate of 1 Gyr has been suggested (Rappaport et al. 2009). The recent discovery of close companion by Gies et al. (2008) to Regulus ${ }^{7}$ has led to consideration of the object's 'huge' quadrupole mass moment $Q$ (Iorio 2008); estimates of $Q$ indicate that detection of the correction to the Keplerian period due to $Q$ would be possible with period measurements precise to $\sim 20$ seconds; however, the current state of the art for the period measurement is about two orders of magnitude beyond that at $P=40.11 \pm 0.02$ days (Gies et al. 2008).

Also as with Altair, Regulus has now been imaged by CHARA-MIRC (Che et al. 2011). The broad details of the earlier McAlister et al. (2005) study have been validated by Che et al. (2011) (Table 1), but further rich detail has been added from the imaging. In particular, a much tighter constraint on inclination is now available $(i=$

\footnotetext{
${ }^{7}$ In addition to its close companion, Regulus has a wide companion $\alpha$ Leo B at a separation of $\sim 175^{\prime \prime}$, also a binary. However, at such a distance, it will not have ever interacted with Regulus (McAlister et al. 2005).
} 
$86.3_{-1.6}^{+1.0}$ ), along with tighter constraints on pole and equator temperature, and further strong evidence of departures from the 'standard' von Zeipel gravity darkening, with $\beta=0.188_{-0.029}^{+0.012}$.

\subsection{Alderamin $(\alpha$ Cep)}

A second CHARA Array commissioning target, Alderamin ( $\alpha$ Cep, HR 8162, HD 203280; $\left.\alpha=21^{\mathrm{h}} 18^{\mathrm{m}} 34.77^{\mathrm{s}}, \delta=+62^{\circ} 35^{\mathrm{m}} 08.06^{\mathrm{s}}\right)$, was also observed with the array, also benefiting from the large amount of $\{u, v\}$ coverage afforded by the facility's multiple baselines (van Belle et al. 2006). Alderamin is an A7IV star (Johnson and Morgan 1953), making it an attractive observing candidate given its similarities to Altair. Alderamin is $3 \times$ further than Altair but, strangely, at roughly the same X-ray luminosity (Robrade and Schmitt 2009); this suggests that coronal X-ray emission sets in at about A7 for main sequence stars (at least at the sensitivity levels of current instrumentation). Alderamin has been known to be a rapid rotator for many decades (Slettebak 1955), with spectroscopic estimates rotational velocity that range from $v \sin i=180-200 \mathrm{~km} \mathrm{~s}^{-1}$ (Gray 1980; Abt and Morrell 1995) to 245-265 $\mathrm{km} \mathrm{s}^{-1}$ (Abt and Moyd 1973; Bernacca and Perinotto 1970). This object was recently reobserved (Zhao et al. 2009) with the array using the newer multi-way MIRC combiner, which not only provided visibility amplitude data on baseline pairs but closure phase data from baseline triplets.

Both the van Belle et al. (2006) and Zhao et al. (2009) investigations attempted to fully characterize the fundamental parameters associated with the rapidly rotating stellar photosphere, including inclination $i$, sky position angle $\alpha$, polar and equatorial radii and temperatures, and most significantly, gravity darkening $\beta$. As noted in Sect. 3.2, a single value of $\beta$ is perhaps an incomplete characterization of the true physical nature of the star's surface, particularly given the range of temperatures, from $T_{\text {pole }}=8600 \pm 300 \mathrm{~K}$ to $T_{\text {equator }}=6600 \pm 200 \mathrm{~K}$; following the canonical temperatures of static models found in Cox (2000), we see this ranges from spectral types $\sim \mathrm{A} 3 \mathrm{~V}$ to $\sim \mathrm{F} 5 \mathrm{~V}$, respectively. This range of temperatures brackets the radiativeto-convective transition temperature of $\sim 8300 \mathrm{~K}$ (Christensen-Dalsgaard 2000; Neff and Simon 2008), allowing for equatorial convective activity but no similar polar activity (Rachford and Foight 2009).

Contrasting the results from the earlier van Belle et al. (2006) study with that of Zhao et al. (2009) is instructive in illustrating the power of various interferometric data sets. The former, having only visibility amplitude data available (only the 2-way CHARA Classic combiner was online at the time of the study), came up with an inclination much larger than the latter $\left(88.2_{-13.3}^{+1.8}\right.$ versus $55.70 \pm 6.23$ degrees $)$. Simply put, the former was rather insensitive to the asymmetric stellar surface brightness distribution, which manifests itself in the poor value for $i$ and (probably unreasonably) low value for $\beta$; the latter, using CHARA-MIRC data which produce data sets not just with size-sensitive visibility amplitude data, but also with asymmetry-sensitive closure phase data, was able to produce a significantly more precise-and presumably accurate-values for $i$ and $\beta$. This in turn affected the entire ensemble of the values in the solution set; it is interesting that the best value for gravity darkening still deviates from the 'von Zeipel ideal' with $\beta=0.216 \pm 0.021$. 
It is also noteworthy that the similar visibility-only solution for Regulus in McAlister et al. (2005) also found a lower value for $\beta$ than the spectroscopy/visibility solution found in the same paper $(\beta=0.13 \pm 0.05$ versus $0.25 \pm 0.11)$. Investigators may wish to consider if such data sets are insufficiently rich to providing constraints upon the stellar surface morphology to characterize gravity darkening: an excellent example of this can be seen in Fig. 12 of Zhao et al. (2009), where for certain onsky orientations, degeneracies may form between various parameters (in this case, $\beta$ and $i$, when $i$ is large). In particular, for those investigators wishing to establish interferometry-only data sets to determine absolute rotation velocities for comparison with spectroscopic $v \sin i$ values, closure phase characterizations of asymmetric surface bright distributions will be highly sensitive to rotation axis inclination values.

Extending the implications of the observations, in van Belle et al. (2006), a simple analysis of the rotation history of the star via the simple application of conservation of angular momentum was considered, and implied that its present fractional breakup velocity $\left(v_{e} / v_{c}=0.83 \pm 0.05\right.$ - similar to the Zhao et al. (2009) value, so this line of reasoning is unaffected by the poor values of $i$ and $\beta$ in that study) would have been even higher in its zero-age main sequence history, with $v_{e} / v_{c} \simeq 0.92-0.98$. Evidence of such an large earlier rotation rate has significant implications for the star's formation history.

In their study of the onset of convective zones in main sequence stars, both Altair and Alderamin are cited by Neff and Simon (2008) as cases that demonstrate the lack of fidelity in categorizing stars solely by spectral type or mass. These same investigators found no FUSE UV evidence for deepening of convective zones due to the rapid rotation of these objects, as suggested not only by the interferometry data but also by the models of MacGregor et al. (2007).

\subsection{Vega $(\alpha$ Lyr $)$}

Vega ( $\alpha$ Lyr, HR7001, HD172167; $\alpha=18^{\mathrm{h}} 36^{\mathrm{m}} 56.34^{\mathrm{s}}, \delta=+38^{\circ} 47^{\mathrm{m}} 01.29^{\mathrm{s}}$ ) is a particularly interesting special case of an observed rapid rotator in that it is viewed nearly pole-on relative to the other examples cited thus far. The long-standing use of Vega as a spectrophotometric standard (e.g., Hayes 1967; Straizys et al. 1976) makes understanding the surface brightness distribution of this object at the highest levels of detail more than an abstract scientific exercise, and one of fundamental utility. The discovery of Vega's debris disk (Aumann et al. 1984) due to its mid-infrared excess flux called into question its validity as a fundamental standard. This situation has been further complicated by the fact that the star is a rapid rotator, as first suggested by Gray (1988). This result, based upon detailed spectroscopic line modeling, has been confirmed by interferometry, as we shall see below.

As with Altair and Regulus, Hanbury Brown et al. (1974) observed Vega and obtained a limb-darkened angular size of $3.24 \pm 0.07$ mas (and a uniform-disk size of $3.08 \pm 0.07$ mas) through a $443 \pm 5 \mathrm{~nm}$ filter. As with all of the objects in this review, the intervening generation of optical interferometer prototypes after NIIGI2T, IRMA, IOTA (to name a few) — did not observe Vega as a scientific target, due to limited spatial resolution. Further scientifically significant data from interferometer on this object came with the longer baselines of PTI when Ciardi et al. (2001) 
observed it and found puzzling signs of residuals in the $K$-band fits, consistent with a debris disk signal contaminating the stellar photospheric signal (as discussed in more detail in Barnes 2009; Lawler et al. 2009; Akeson et al. 2009). This finding was consistent with the further investigations with the CHARA Array (Absil et al. 2006) and IOTA (Defrère et al. 2011). These PTI and CHARA Array interferometric studies set the stage for two further, more detailed, studies of the star's photosphere itself.

Both NOI (Peterson et al. 2006b) and the CHARA Array (Aufdenberg et al. 2006) published studies of Vega early in 2006, in the visible ( 500-800 nm) and $K^{\prime}$-band, respectively. Both studies found strong evidence for rapid rotation in a nearly pole-on star, arriving at remarkable agreement in inclination $\left(4.54 \pm 0.33^{\circ}\right.$ and $4.7 \pm 0.3$ ), equatorial rotational velocity ( $274 \pm 14$ and $270 \pm 15)$, and other parameters (see Table 3) despite completely independent development of their methodologies and differing observational wavelengths. Agreement between these two disparate approaches is reassuring; however, further investigations by the NOI group (Yoon et al. 2008, 2010) indicate a lower rotation speed $\left(v_{\mathrm{eq}}=175 \pm 33\right)$ coupled with lower than previously expected mass and sub-solar metallicity $\left(2.14 \pm 0.08 M_{\odot}\right.$, $Z=0.0080 \pm 0.0033)$.

Interferometric results indicating a 'fast' rotation speed of $\approx 270 \mathrm{~km} \mathrm{~s}^{-1}$ are disputed in the spectroscopic analysis by Takeda et al. (2008), who favor a more 'moderate' speed of $\approx 175 \mathrm{~km} \mathrm{~s}^{-1}$. However, using the parameters from Peterson et al. (2006b), Yoon et al. (2008) evaluate ELODIE archival spectra of Vega and invoke turbulence on large scales ('cyclones', even, to use their language) in their analysis to achieve a match between the data and their calculated spectral lines. The implications of such a result are intriguing, to say the least: these results are themselves rather dramatic, but the underlying approach of interferometric observations to guide spectroscopic analysis could be a far-reaching in application to more than just studies of rapidly rotating stars.

It is interesting to note that there has been no detection of X-rays by CHANDRA even after $29 \mathrm{ks}$ of observing (Pease et al. 2006), even though (as noted above) they have been detected for Altair and Alderamin (Robrade and Schmitt 2009). The overall set of 'problems' with Vega are reviewed in the article by Gray (2007), of which the interferometrically detected phenomena of IR excess and rapid rotation play a large role. Ultimately, all of these various pathologies may lead astronomers to use stars other than Vega for photometric standards, as already proposed by Engelke et al. (2010).

\subsection{Rasalhague ( $\alpha \mathrm{Oph})$}

Rasalhague $\left(\alpha\right.$ Oph A, HR6556, HD 159561; $\alpha=17^{\mathrm{h}} 34^{\mathrm{m}} 56.07^{\mathrm{s}}, \delta=$ $+12^{\circ} 33^{\mathrm{m}} 36.13^{\mathrm{s}}$ ), due to a number of unique circumstances, is a particularly interesting rapid rotator and is quickly becoming a fundamental laboratory for exploring physics of rapid rotation. As with many of these objects, it was originally observed interferometrically by Hanbury Brown et al. (1974).

An A5IV (Gray et al. 2001) $\delta$ Scuti variable star, $\alpha$ Oph A has been observed to be a nearly edge-on rapid rotator with $\omega / \omega_{c} \sim 0.88$ with the imaging study of 


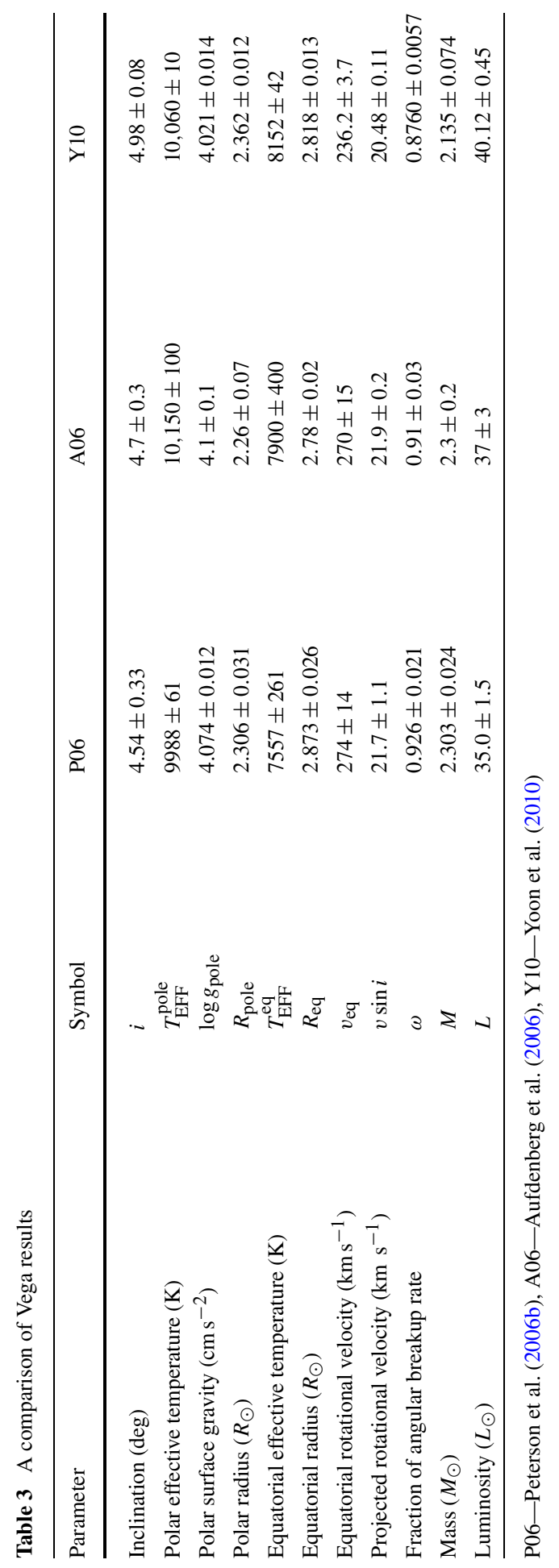


Zhao et al. (2009). As noted above in the discussion for Alderamin (Sect. 5.4), the edge-on orientation makes solving for $\beta$ problematic, so Zhao et al. (2009) proceeded with a fixed value of $\beta=0.25$. Monnier et al. (2010b) observed $\alpha$ Oph A with the MOST astroseismology satellite, noting that this inclination is particularly favorable (odd-parity $l-|m|$ modes are suppressed, simplifying mode identifications) and detected rotationally modulated $g$-modes in the object. The (unexpected) linear relationships between $g$-mode spacings were provisionally explained as dispersion-free Kelvin waves. Hinkley et al. (2011) present an improved astrometric orbit of $\alpha$ Oph $\mathrm{A}$ and $\mathrm{B}$ from eight years of AO imaging data, and point out that a significantly improved determination of the 7.9-year astrometric orbit will be possible during the $\sim 50$ mas April 2012 periastron, using optical interferometry. This in turn will permit mass determination of $\alpha$ Oph A at the few percent level. Collectively, these studies allow Rasalhague to be a prototype for probing the physics of stellar interiors, and understanding the effects of rapid rotation in that regime.

\section{$5.7 \beta$ Cas (Caph)}

The most recent addition to the stable of rapid rotators observed with optical interferometry is $\beta$ Cas (Che et al. 2011). Its spectral type of F2III-IV (Rhee et al. 2007) indicates it is a 'retired' A-type star, evolving off the main sequence, and makes this observation a noteworthy one, in that it is the first object that is not (currently) ' $\mathrm{A}$ ' or ' $\mathrm{B}$ ' spectral type. As a cooler object, it represents an expansion of the tests derived from this technique into new areas of discovery space. The rotational velocity of $\sim 70 \mathrm{~km} \mathrm{~s}^{-1}$ (Rachford and Foight 2009) did not, on its face, give an a priori suggestion that it was an rapid rotator, although the small value of $P / \sin i$ reported therein $\left(2.48^{\mathrm{d}}\right)$ certainly hinted at the possibility.

Observationally, $\beta$ Cas presents itself nearly pole-on, with $i=19.9 \pm 1.9^{\circ}$, indicating a significant rotation rate $\left(v=206 \mathrm{~km} \mathrm{~s}^{-1}, P=1.12_{-0.04}^{+0.03}\right.$ days). As with

Regulus, Che et al. (2011) also fit for $\beta$, finding a value of $0.146_{-0.007}^{+0.013}$. This lower value is consistent with trends toward a lower expected value for a star with lower surface temperature (see Fig. 9 of Che et al. 2011, and Claret 2000), although it is not nearly as small as would be expected for a fully radiative photosphere $(\beta=0.08)$.

\section{Broad impact in astronomy}

Although there is only a small number of results thus far, there are already broad and significant impacts throughout astronomy as the implications of such detailed new views of stars diffuse through the field. For example, the particulars of the analysis of the spectral energy distribution of Vega already noted above (Aufdenberg et al. 2006) significantly revises our view of this fundamental standard (Rieke et al. 2008).

The interaction between spectroscopy and rapid rotator analysis has a long history, notably from the work by Collins $(1963,1965)$, Collins and Harrington (1966). The interferometric observations have begun to guide spectroscopy-for example, 
discrepancies in $T_{\mathrm{EFF}}$ indicated in $\mathrm{H} \alpha$ and $\mathrm{H} \beta$ Balmer line profiles in Altair found in Smalley et al. (2002) and Smalley and Kupka (2003) are resolved when the lower effective temperatures indicated by interferometry are used. Similarly, as predicted in Smalley et al. (2002), subsequent observations of Rasalhague also indicate a lower average $T_{\mathrm{EFF}}$, particularly given the edge-on orientation of this object, which resolve similar temperature anomalies. Such agreements are contrasted with the example of $v \sin i$ estimates for Vega, which even when account for pole-on rapid rotation (Hill et al. 2004) fall short of the true magnitude of the star's extreme rotational speed (Aufdenberg et al. 2007). Similarly, a line profile analysis of Altair incorporating not just the Balmer lines but a larger 650-line list (Reiners and Royer 2004) does not quite fall into agreement is the most recent values for inclination and rotation speed (Monnier et al. 2007). Analyses are now being forced to cast a wider net and consider such things as revised metallicities (Yoon et al. 2010) in order to reconcile spectroscopy and interferometry, a move which perhaps mirrors other upheavals in astronomy related to metallicity (Asplund et al. 2009).

Folding details of rapid rotation from these observations into spectroscopic modeling has not been prevented by these challenges, however. The line profiles of Huang and Gies (2006) are built using hemispheric averages for $\left\{T_{\mathrm{EFF}}, \log g, v \sin i\right\}$ in their analysis using line profiles to investigate evolution of stellar rotation in young clusters. Neff and Simon (2008) examined O VI emission lines in detail in the context of the rapid rotation reported for Altair and Alderamin, to explore the onset of convection zones as made possible by rotational equatorial cooling - their detection of such emission in a variety of stars indicates that magnetic activity without substantial convective zones needs to be possible in current models. Khalack (2005) also point out that magnetic dipole strength can be overestimated if stellar oblateness is not taken into account. Combining interferometric and spectroscopic observations is suggested as a way to probe the properties of the rotation law of the external layers of rapidly rotating stars (Zorec et al. 2011).

Underlying spectroscopy, observational characterization of rapid rotation has also started to make significant inroads with stellar modeling (Domiciano de Souza et al. 2002). Comparison of the results of observed rapid rotators to stellar models is often difficult, in that typical models are constructed for non-rotating stars. Che et al. (2011) take the approach of applying corrections from predictions such as Sackmann (1970) to facilitate comparison to $Y^{2}$ models (Yi et al. 2001), with some success, although they note further work is warranted.

From an examination of collective results to date, Che et al. (2011) suggests that for general use in modeling rapidly rotating stars, a gravity darkening value of $\beta=0.19$ is more appropriate. The interferometric results seem to validate the suggestion in Tassoul (2000) that solid-body rotation is impossible for a rapid rotator: temperature and pressure consistency across the stellar surface is disrupted by such rotation. This disruption leads to temperature and pressure gradients between the poles and equator, which in turn induces meridional circulation and breaks down strict radiative equilibrium. The differential angular momentum of the meridional circulation's matter flow — the higher latitude material carries less angular momentum-will then cause differential rotation.

Stellar rotation affects abundance, mass loss rates, and overall evolution (for a discussion of the implications for the most massive stars, see the 13-part series that be- 
gins with Meynet and Maeder 1997). New grids are in the process of being produced which incorporate the observational interferometric results, including smoother transition values for gravity darkening (Claret 2005). Alternatively, the basic parameters being discovered through interferometry are being used as anchor points in stellar evolution models to 'run the clock backwards', and explore the history of parameters such as angular momentum (van Belle et al. 2006).

Beyond of spectroscopy and modeling, there are a broad number of areas where these observational results are being considered as noteworthy. The naturally complementary techniques of asteroseismology and interferometry are seen as deeply connected on the point of rotation by Cunha et al. (2007) and Aerts et al. (2009); observation extension of these two techniques in a conjoined fashion has already been demonstrated in Monnier et al. (2010b)

In considering transiting extrasolar planets, Seager and Hui (2002) noted the stellar oblateness measured by van Belle et al. (2001), and uses that as a starting point to discuss constraining the rotation rate of those planets through measurements of the planetary oblateness. Such measurements are expected to be possible with the high-precision data being produced by the Kepler mission. Barnes (2009) notes that the Rossiter-McLaughlin measurements that have been employed to date to probe the spin-orbit alignments of transiting systems are difficult about rapid rotators, but suggests that the unusual and distinctive transiting lightcurves associated with such systems could instead be utilized to explore those alignments.

Evaluation of rapid rotation is seen as a necessary part of understanding microlensing signals (Han and Chang 2006), with shapes of caustics being affected by source oblateness; Rattenbury et al. (2005) attempt to derive from microlensing event photometry the shape of the source star. Space climate is seen as directly connected to the irradiance properties of our sun, which in turn are connected to its shape (Lefebvre et al. 2007; Lefebvre et al. 2005). On a fundamental level, measurement of stellar oblateness is now acknowledged as one of the basic techniques for measurement of stellar rotation (Royer 2005).

\section{Future target list}

The seminal work of Hanbury Brown et al. (1974) providing angular diameter sizes on 32 bright, hot, nearby stars may be reviewed in considering the importance of expanding our knowledge base of rapidly rotating stars. The error estimates on the limb-darkened angular diameters presented in this paper ranged from $2 \%-14 \%$, with a median value of $6.3 \%$. Cross-referencing these results against the rotational velocities readily found in the catalog of Glebocki and Gnacinski (2005), we find 13 objects in common. We can estimate average linear radii $R$ and mass $M$ from the appropriate sections in Cox (2000), and thereby estimate oblateness using the prescription found in the appendix of van Belle et al. (2006).

What is instructive is to not consider the overall degree of oblateness predicted for these 13 objects, but to compare that degree of non-sphericity with the error estimates of spherical size given in Hanbury Brown et al. (1974). In two cases ( $\alpha$ Aql, $\alpha$ Eri), the predicted oblateness is significantly in excess $(\sim 2-3 \times$ greater $)$ than the 
spherical size quoted error; for three objects ( $\beta$ Car, $\zeta$ Pup, $\epsilon$ Car) oblateness is comparable $(\sim 0.9-1.0 \times)$ to the quoted error, with a fourth also being reasonably large in comparison ( $\alpha$ Vir, at $0.6 \times$ ). Better than $45 \%$ of the objects available for such a line of inquiry show strong motivation for developing a deeper understanding of their true size, particularly since the Hanbury Brown et al. (1974) values have propagated widely in the literature.

To date, only 7 targets have been investigated with optical/near-infrared interferometry, which may leave the impression that a substantially larger sample is beyond the capabilities of current facilities. This is simply not true. To probe the possibilities of this manner of study, we created a list of targets that are appealing to presentday facilities. Our assumptions were modest: angular sizes in excess of 0.50 mas (roughly the current limiting angular size of modern optical interferometers), and size ratios in excess of $R_{b} / R_{a}-1>0.05$ (as dictated by the visibility precisions achievable with those facilities.) Starting with the rotational velocity catalog of Glebocki and Gnacinski (2005), we cross-referenced it against Hipparcos and 2MASS (Perryman et al. 1997; Cutri et al. 2003) to establish $V$ and $K$ magnitudes. From these values, using the rough angular size predictor found in van Belle (1999), we estimated angular sizes and cut those stars with $<0.50$ mas. Using the quoted spectral types of Hipparcos to estimate average linear radius $R$ and mass $M$ from the nominal values found in Cox (2000), we then were able to estimate oblateness, cutting for $R_{b} / R_{a}-1<0.05$.

The resultant catalog of 354 objects is illustrated in Fig. 13, of which almost a third are 'Altair-class' rotators with $R_{b} / R_{a}-1>0.15$; the complete catalog is provided in an Appendix. This catalog is, of course, incomplete, due to the imperfection of the assumptions involved, and it further reflects the incompleteness of Glebocki and Gnacinski (2005) (although the rather uniform sky distribution suggests this degree of incompleteness is low-e.g. no bias of northern hemisphere targets over those in the south). This approach also completely misses low-inclination rapid rotators with low values of $v \sin i$, as illustrated in the case of Caph (Sect. 5.7). The limitations on use of $v \sin i$ as a predictive parameter for oblateness is clearly seen in Fig. 13: each of the targets that has been observed to date has an actual value in excess (in some cases, well in excess) of the prediction from $v \sin i$. This of course illustrates the impact of the $\sin i$ term-and portends many fruitful future observations of oblateness with interferometry!

\section{Conclusion}

Direct observations of rapid rotators by long-baseline interferometers have matured rapidly over the past 10 years. Simple parametric models have given way to detailed models that probe the underlying stellar structure, and stunning images of photospheres that confirm the input physics in a dramatic, model-independent fashion (Fig. 14). The lessons learned and confidence collectively gained by the specialists carrying out these investigations is now being applied to a greater variety of objects. As the observed sample is expanded to objects of lower temperatures, some surprises are expected and have already been found (Che et al. 2011). 


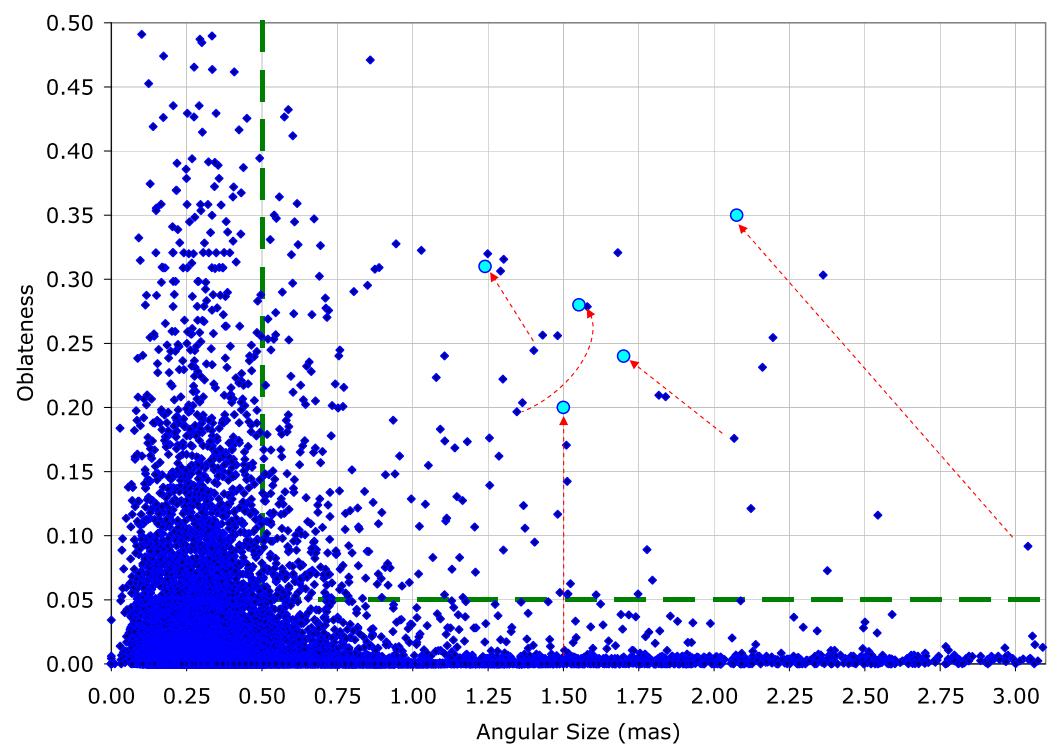

Fig. 13 Illustration of prospective targets as outlined in Sect. 7. Targets with estimates of $\theta>0.50$ mas and oblateness $R_{b} / R_{a}-1>0.05$ are in the 'allowed' region to the upper right of the dashed line. Actual values for observed targets are shown in relationship to their proxy values via a dotted line. Of particular interest is the clear indication that actual oblateness values are always well in excess of the simple predictions from $v \sin i$ values

Matching this expansion of target selection has been a growth of instrumental capabilities. The 4-telescope CHARA-MIRC instrumentation that provided the first images (Monnier et al. 2007) is being expanded to full 6-way combination with sensitivity improvements through external fringe tracking (Monnier et al. 2010a); the first results from 4-telescope beam combination at VLTI with the PIONIER visitor instrument are very impressive (Berger et al. 2010); a new NOI combiner ('VISION') will add true 6-way combination to this facility as well and is slated to be on-sky in mid-2012. All of these developments bode well for further investigation into rapid rotators, possibly with complementary data sets spanning from the $500 \mathrm{~nm}$ through $2.4 \mu \mathrm{m}$, along with to increased 'snapshot' capability from the richer data sets.

What has been especially gratifying to see has been the rapid ingestion of these results into a wide variety of other endeavors in the field (Sect. 6). This has taken this activity from being just a 'cottage industry' to being one of deep implications for many of the foundational ideas in astronomy.

Acknowledgements The author would like to gratefully acknowledge useful discussions with Doug Gies during the preparation of this manuscript. Portions of this manuscript were prepared while the author was in residence at the European Southern Observatory (ESO). This work made use of the SIMBAD reference database, the VizieR catalogue access tool, and the NASA Astrophysics Data System, all of which are indispensable resources for astronomy. Funding for this research has been provided in part by Lowell Observatory. 

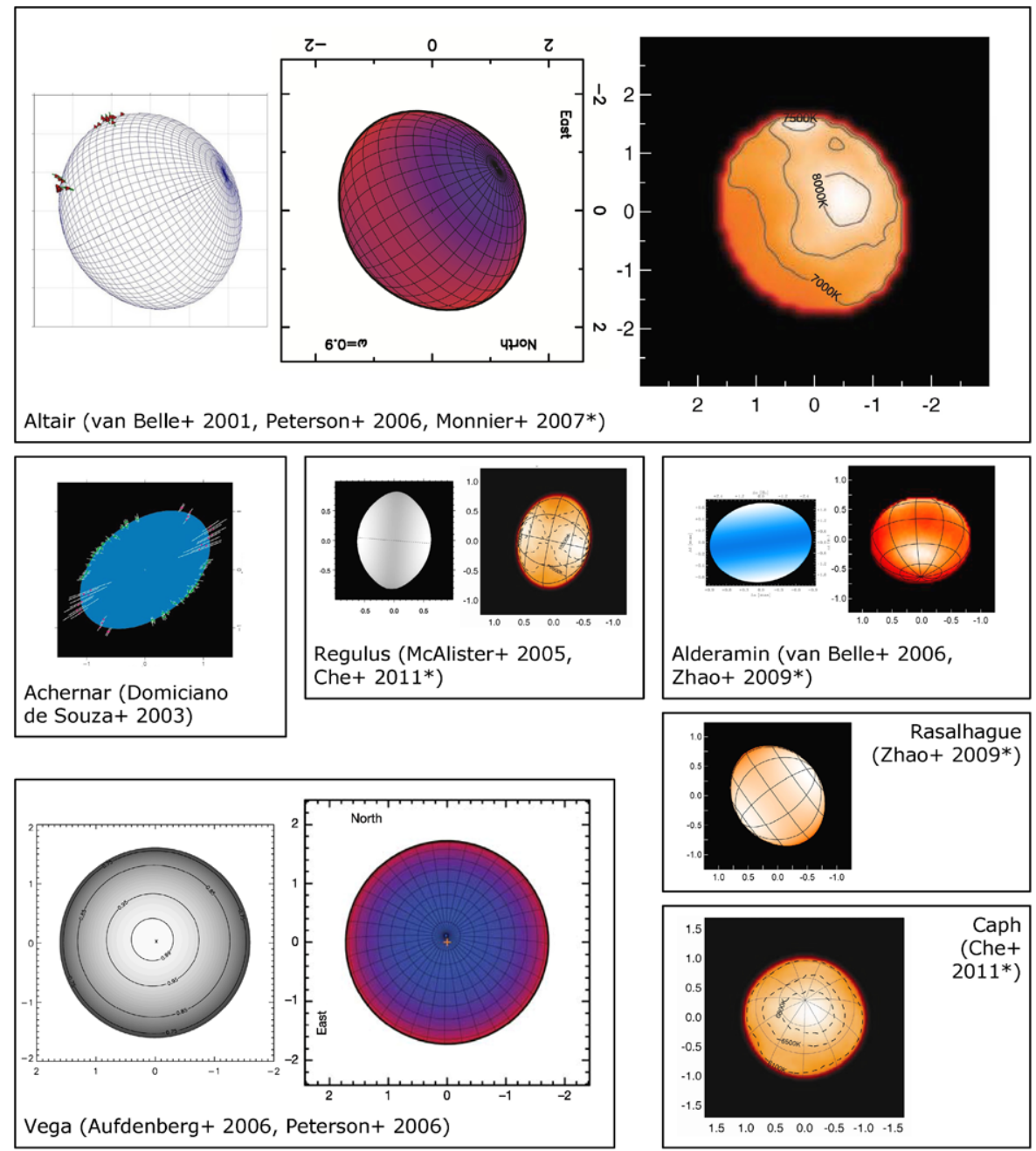

Regulus (McAlister+ 2005, de Souza+ 2003)

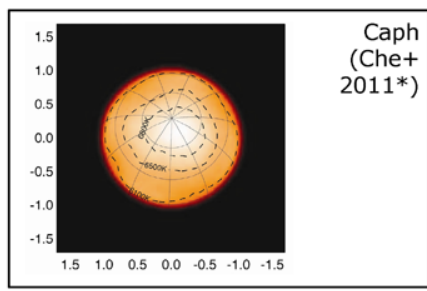

All sizes to the same scale of 1 mas $=2.6 "$

* denotes actual image

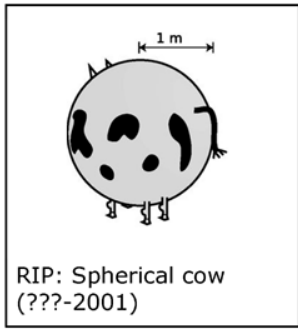

Fig. 14 Illustration of figures/images of rapid rotators produced over the past 10 years by PTI, NOI, the CHARA Array, and VLTI. All illustrations are normalized to the same page scale-relative apparent sizes are correct 
Open Access This article is distributed under the terms of the Creative Commons Attribution License which permits any use, distribution, and reproduction in any medium, provided the original author(s) and the source are credited.

\section{Appendix: Prospective future targets}

Table 4 Summary of possible future targets for interferometric studies of rapid rotators as described in Sect. 7; angular size and oblateness values here are estimates

\begin{tabular}{|c|c|c|c|c|c|c|c|c|}
\hline HD & $\begin{array}{l}\text { RA } \\
\text { hh:mm:ss }\end{array}$ & $\begin{array}{l}\text { DE } \\
\text { dd:mm:ss }\end{array}$ & $\begin{array}{l}\text { Spectral } \\
\text { type }\end{array}$ & $\begin{array}{l}V \\
(\mathrm{mag})\end{array}$ & $\begin{array}{l}K \\
(\mathrm{mag})\end{array}$ & $\begin{array}{l}v \sin i \\
(\mathrm{~km} / \mathrm{s})\end{array}$ & $\begin{array}{l}\theta_{\mathrm{EST}} \\
(\mathrm{mas})\end{array}$ & $\begin{array}{l}\text { Oblateness } \\
R_{b} / R_{a}-1\end{array}$ \\
\hline 225003 & 000229.76 & +08 2908.1 & F0V & 5.70 & 4.91 & 165 & 0.51 & 0.06 \\
\hline 225132 & 000344.37 & -172009.5 & B9IVn & 4.55 & 4.56 & 190 & 0.57 & 0.12 \\
\hline 360 & 000817.55 & -084926.5 & G8III: & 5.99 & 3.75 & 240 & 0.94 & 0.19 \\
\hline 493 & 000921.02 & -275916.5 & F3V & 5.42 & 4.38 & 170 & 0.65 & 0.07 \\
\hline 2262 & 002612.12 & -434047.7 & A7V & 3.93 & 3.59 & 245 & 0.91 & 0.15 \\
\hline 2696 & 003022.67 & -234715.8 & A3V & 5.17 & 4.83 & 150 & 0.51 & 0.05 \\
\hline 2884 & 003132.56 & -625729.1 & B9V & 4.36 & 4.48 & 170 & 0.59 & 0.06 \\
\hline 3283 & 003627.34 & +601934.4 & A4III & 5.78 & 4.79 & 100 & 0.54 & 0.07 \\
\hline 4180 & 004443.50 & +481703.8 & B5III & 4.48 & 4.40 & 255 & 0.62 & 0.36 \\
\hline 5394 & 005642.50 & +604300.3 & B0IV:evar & 2.15 & 1.76 & 260 & 2.12 & 0.12 \\
\hline 6903 & 010949.20 & +193930.2 & G0III & 5.57 & 4.18 & 95 & 0.73 & 0.06 \\
\hline 7344 & 011343.80 & +073431.8 & A7IV & 5.21 & 4.57 & 265 & 0.59 & 0.25 \\
\hline 7788 & 011545.50 & -685234.5 & F6IV & 4.25 & 3.88 & 135 & 0.80 & 0.09 \\
\hline 9352 & 013325.71 & +581938.4 & $\mathrm{KOIb}+\cdots$ & 5.69 & 1.77 & 50 & 2.54 & 0.12 \\
\hline 10144 & 013742.75 & -571412.0 & $\mathrm{~B} 3 \mathrm{Vp}$ & 0.45 & 0.88 & 250 & 3.04 & 0.09 \\
\hline 10148 & 013851.71 & -211631.7 & F0V & 5.58 & 4.75 & 170 & 0.55 & 0.06 \\
\hline 10516 & 014339.62 & +504119.6 & B2Vpe & 4.01 & 3.71 & 505 & 0.86 & 0.47 \\
\hline 10830 & 014538.65 & -250308.8 & F2IV & 5.29 & 4.46 & 120 & 0.62 & 0.06 \\
\hline 13041 & 020829.15 & +375133.1 & A5IV-V & 4.78 & 4.43 & 135 & 0.62 & 0.06 \\
\hline 13174 & 020925.29 & +255623.9 & F2III & 4.98 & 4.06 & 160 & 0.75 & 0.24 \\
\hline 14228 & 021630.50 & -513043.6 & B8IV-V & 3.56 & 4.13 & 250 & 0.68 & 0.17 \\
\hline 14055 & 021718.84 & +335050.4 & A1Vnn & 4.03 & 3.96 & 240 & 0.76 & 0.13 \\
\hline 15008 & 022145.02 & -683933.9 & A3V & 4.08 & 3.96 & 180 & 0.76 & 0.07 \\
\hline 14690 & 022212.41 & -005305.1 & F0Vn & 5.42 & 4.58 & 185 & 0.59 & 0.08 \\
\hline 15233 & 022453.99 & -601841.9 & F2III & 5.36 & 4.42 & 140 & 0.64 & 0.17 \\
\hline 15130 & 022557.01 & -121725.6 & $\mathrm{~A} 0 \mathrm{~V}$ & 4.88 & 4.81 & 210 & 0.51 & 0.10 \\
\hline 15257 & 022809.99 & +294010.3 & F0III & 5.29 & 4.59 & 85 & 0.59 & 0.06 \\
\hline 236970 & 023318.35 & +561905.0 & A2Iab & 8.84 & 4.90 & 170 & 0.60 & 0.30 \\
\hline 16555 & 023724.26 & -523235.1 & $\mathrm{~A} 6 \mathrm{~V}$ & 5.30 & 4.53 & 315 & 0.61 & 0.27 \\
\hline 16978 & 023935.22 & -681601.0 & B9III & 4.12 & 4.25 & 100 & 0.65 & 0.05 \\
\hline 16754 & 023947.92 & -425329.9 & $\mathrm{~A} 2 \mathrm{~V}$ & 4.74 & 4.46 & 245 & 0.61 & 0.14 \\
\hline 16970 & 024318.12 & +031410.2 & $\mathrm{~A} 3 \mathrm{~V}$ & 3.47 & 3.08 & 190 & 1.16 & 0.08 \\
\hline
\end{tabular}


Table 4 (Continued)

\begin{tabular}{|c|c|c|c|c|c|c|c|c|}
\hline HD & $\begin{array}{l}\text { RA } \\
\text { hh:mm:ss }\end{array}$ & $\begin{array}{l}\text { DE } \\
\text { dd:mm:ss }\end{array}$ & $\begin{array}{l}\text { Spectral } \\
\text { type }\end{array}$ & $\begin{array}{l}V \\
\text { (mag) }\end{array}$ & $\begin{array}{l}K \\
(\mathrm{mag})\end{array}$ & $\begin{array}{l}v \sin i \\
(\mathrm{~km} / \mathrm{s})\end{array}$ & $\begin{array}{l}\theta_{\mathrm{EST}} \\
(\mathrm{mas})\end{array}$ & $\begin{array}{l}\text { Oblateness } \\
R_{b} / R_{a}-1\end{array}$ \\
\hline 17566 & 024532.53 & -673700.2 & A2IV/V & 4.83 & 4.60 & 135 & 0.57 & 0.05 \\
\hline 17573 & 024958.99 & +271538.8 & $\mathrm{~B} 8 \mathrm{Vn}$ & 3.61 & 3.86 & 240 & 0.78 & 0.12 \\
\hline 17584 & 025034.91 & +381908.1 & F2III & 4.22 & 3.24 & 160 & 1.11 & 0.24 \\
\hline 18331 & 025637.45 & -034244.0 & $\mathrm{~A} 3 \mathrm{Vn}$ & 5.16 & 4.86 & 255 & 0.51 & 0.16 \\
\hline 18411 & 025845.65 & +393946.2 & $\mathrm{~A} 2 \mathrm{Vn}$ & 4.68 & 4.44 & 170 & 0.61 & 0.06 \\
\hline 18866 & 025847.77 & -640416.7 & A5III & 4.98 & 4.62 & 115 & 0.57 & 0.10 \\
\hline 18978 & 030223.59 & -233727.6 & $\mathrm{~A} 4 \mathrm{~V}$ & 4.08 & 3.57 & 180 & 0.92 & 0.07 \\
\hline 19319 & 030336.90 & -594415.4 & FOIV & 5.12 & 4.28 & 130 & 0.68 & 0.07 \\
\hline 19107 & 030416.48 & -073603.2 & A8V & 5.26 & 4.74 & 150 & 0.54 & 0.05 \\
\hline 20313 & 030731.90 & -785921.9 & F2II-III & 5.67 & 4.94 & 75 & 0.50 & 0.05 \\
\hline 19275 & 031156.24 & +742337.9 & A2Vnn & 4.85 & 4.71 & 250 & 0.54 & 0.15 \\
\hline 20121 & 031225.68 & -442510.8 & $\mathrm{~F} 3 \mathrm{~V}+\cdots$ & 5.92 & 4.83 & 195 & 0.54 & 0.10 \\
\hline 22192 & 033629.36 & +481133.7 & B5Ve & 4.32 & 4.11 & 375 & 0.71 & 0.28 \\
\hline 22928 & 034255.48 & +474715.6 & B5III SB & 3.01 & 3.26 & 245 & 1.03 & 0.32 \\
\hline 23302 & 034452.52 & +240648.4 & B6III & 3.72 & 3.92 & 215 & 0.76 & 0.24 \\
\hline 23480 & 034619.56 & +235654.5 & B6IV & 4.14 & 4.22 & 285 & 0.67 & 0.27 \\
\hline 23630 & 034729.06 & +240618.9 & B7III & 2.85 & 2.64 & 210 & 1.40 & 0.24 \\
\hline 23850 & 034909.73 & +240312.7 & B8III & 3.62 & 3.88 & 195 & 0.77 & 0.22 \\
\hline 23401 & 035021.48 & +711956.5 & A2IVn & 4.59 & 4.36 & 205 & 0.63 & 0.17 \\
\hline 24554 & 035417.49 & -025717.0 & G8III & 4.46 & 2.45 & 180 & 1.68 & 0.32 \\
\hline 25945 & 040537.30 & -273907.3 & F0IV/V & 5.59 & 4.73 & 135 & 0.55 & 0.05 \\
\hline 25642 & 040635.06 & +502104.9 & A0IVn & 4.25 & 4.15 & 205 & 0.69 & 0.16 \\
\hline 25940 & 040839.67 & +474245.3 & B3Ve & 3.96 & 3.80 & 230 & 0.82 & 0.08 \\
\hline 26612 & 041050.43 & -415937.5 & A9V & 4.93 & 3.95 & 250 & 0.80 & 0.15 \\
\hline 26574 & 041151.93 & -065016.0 & F2II-III & 4.04 & 3.21 & 105 & 1.11 & 0.11 \\
\hline 27901 & 042457.06 & +190231.5 & F4V & 5.97 & 4.98 & 150 & 0.50 & 0.06 \\
\hline 28024 & 042618.39 & +224849.3 & $\mathrm{~A} 8 \mathrm{Vn}$ & 4.28 & 3.76 & 195 & 0.85 & 0.09 \\
\hline 28052 & 042620.67 & +153706.0 & F0V $\ldots$ & 4.48 & 4.03 & 195 & 0.75 & 0.09 \\
\hline 29992 & 044203.45 & -370841.2 & F3V & 5.04 & 4.09 & 185 & 0.75 & 0.09 \\
\hline 30478 & 044421.12 & -594358.2 & A8/A9III/IV & 5.28 & 4.73 & 230 & 0.55 & 0.26 \\
\hline 30211 & 044530.14 & -031516.6 & B5IV & 4.01 & 4.40 & 160 & 0.60 & 0.07 \\
\hline 30739 & 045036.72 & +085400.9 & $\mathrm{~A} 1 \mathrm{Vn}$ & 4.35 & 4.17 & 235 & 0.69 & 0.13 \\
\hline 30780 & 045122.41 & +185023.8 & A7IV-V & 5.08 & 4.49 & 145 & 0.61 & 0.06 \\
\hline 30912 & 045247.09 & +275351.3 & F2IV & 5.97 & 4.98 & 155 & 0.50 & 0.11 \\
\hline 31109 & 045253.68 & -052709.9 & A9IV & 4.36 & 3.72 & 170 & 0.87 & 0.13 \\
\hline 32045 & 045955.71 & -123213.9 & F0V & 4.78 & 4.01 & 185 & 0.77 & 0.08 \\
\hline 33111 & 050751.03 & -050510.5 & A3IIIvar & 2.78 & 2.40 & 190 & 1.58 & 0.28 \\
\hline 32991 & 050755.43 & +214217.4 & $\mathrm{~B} 2 \mathrm{Ve}$ & 5.84 & 4.78 & 220 & 0.55 & 0.06 \\
\hline 33328 & 050908.78 & -084514.7 & B2IVn & 4.25 & 4.71 & 325 & 0.52 & 0.25 \\
\hline 33802 & 051217.89 & -115208.9 & B8V & 4.45 & 4.65 & 195 & 0.54 & 0.07 \\
\hline 34658 & 051911.23 & +023545.4 & F5IIvar & 5.34 & 4.33 & 80 & 0.67 & 0.09 \\
\hline
\end{tabular}


Table 4 (Continued)

\begin{tabular}{|c|c|c|c|c|c|c|c|c|}
\hline HD & $\begin{array}{l}\text { RA } \\
\text { hh:mm:ss }\end{array}$ & $\begin{array}{l}\text { DE } \\
\text { dd:mm:ss }\end{array}$ & $\begin{array}{l}\text { Spectral } \\
\text { type }\end{array}$ & $\begin{array}{l}V \\
(\mathrm{mag})\end{array}$ & $\begin{array}{l}K \\
\text { (mag) }\end{array}$ & $\begin{array}{l}v \sin i \\
(\mathrm{~km} / \mathrm{s})\end{array}$ & $\begin{array}{l}\theta_{\mathrm{EST}} \\
(\mathrm{mas})\end{array}$ & $\begin{array}{l}\text { Oblateness } \\
R_{b} / R_{a}-1\end{array}$ \\
\hline 36705 & 052844.78 & -652656.2 & K1III(p) & 6.88 & 4.69 & 100 & 0.61 & 0.13 \\
\hline 36267 & 053047.05 & +055653.6 & $\mathrm{~B} 5 \mathrm{~V}$ & 4.20 & 4.61 & 180 & 0.55 & 0.05 \\
\hline 36576 & 053331.63 & +183224.8 & B2IV-Ve & 5.67 & 4.78 & 285 & 0.54 & 0.14 \\
\hline 37202 & 053738.68 & +210833.3 & B4IIIp & 2.97 & 2.81 & 320 & 1.29 & 0.31 \\
\hline 37507 & 053853.09 & -071245.8 & $\mathrm{~A} 4 \mathrm{~V}$ & 4.77 & 4.42 & 185 & 0.62 & 0.08 \\
\hline 37490 & 053911.15 & +040717.3 & B3IIIe & 4.50 & 4.81 & 190 & 0.50 & 0.13 \\
\hline 37795 & 053938.94 & -340426.6 & B7IV & 2.65 & 2.83 & 210 & 1.26 & 0.14 \\
\hline 39014 & 054446.42 & -654407.9 & A7V & 4.34 & 3.84 & 225 & 0.82 & 0.12 \\
\hline 38678 & 054657.35 & -144919.0 & A2Vann & 3.55 & 3.29 & 230 & 1.04 & 0.12 \\
\hline 40248 & 055620.94 & -312256.8 & F2III & 5.52 & 4.43 & 110 & 0.64 & 0.10 \\
\hline 41335 & 060413.50 & -064232.2 & B2Vne+ & 5.19 & 4.78 & 445 & 0.53 & 0.33 \\
\hline 41695 & 060609.33 & -145607.0 & $\mathrm{~A} 0 \mathrm{~V}$ & 4.67 & 4.52 & 215 & 0.59 & 0.10 \\
\hline 42818 & 061850.78 & +691912.1 & A0Vn & 4.76 & 4.67 & 325 & 0.55 & 0.27 \\
\hline 44769 & 062346.10 & +043534.2 & A5IV & 4.39 & 3.92 & 125 & 0.79 & 0.06 \\
\hline 45725 & 062849.07 & -070159.0 & $\mathrm{~B} 3 \mathrm{Ve}$ & 3.76 & 4.08 & 325 & 0.70 & 0.17 \\
\hline 45542 & 062857.79 & +201243.8 & B6III & 4.13 & 4.35 & 149 & 0.62 & 0.10 \\
\hline 46273 & 062949.13 & -501420.3 & $\mathrm{~F} 2 \mathrm{~V}$ & 5.28 & 4.33 & 175 & 0.67 & 0.07 \\
\hline 45910 & 063032.94 & +055201.2 & B2:IIIpshev & 6.70 & 4.41 & 430 & 0.69 & 0.30 \\
\hline 46304 & 063223.13 & -055207.4 & $\mathrm{~F} 0 \mathrm{Vnn}+\cdots$ & 5.60 & 4.91 & 200 & 0.51 & 0.09 \\
\hline 46933 & 063503.38 & -225753.4 & A0III & 4.54 & 4.50 & 145 & 0.59 & 0.12 \\
\hline 47670 & 063745.67 & -431145.3 & B8III SB & 3.17 & 3.56 & 225 & 0.89 & 0.31 \\
\hline 50506 & 064002.91 & -804849.4 & A5III & 5.61 & 4.94 & 200 & 0.50 & 0.26 \\
\hline 48917 & 064428.47 & -310413.9 & B2V & 5.23 & 4.89 & 200 & 0.50 & 0.05 \\
\hline 50241 & 064811.54 & -615631.1 & A7IV & 3.24 & 2.57 & 230 & 1.48 & 0.26 \\
\hline 50013 & 064950.47 & -323030.6 & B1.5IVne & 3.50 & 3.55 & 200 & 0.91 & 0.08 \\
\hline 50019 & 065247.34 & +335740.9 & A3III & 3.60 & 3.16 & 130 & 1.11 & 0.11 \\
\hline 51199 & 065537.40 & -200811.7 & F2IV/V & 4.66 & 3.95 & 145 & 0.79 & 0.07 \\
\hline 50973 & 065737.12 & +450538.8 & $\mathrm{A} 2 \mathrm{Vn}$ & 4.90 & 4.79 & 210 & 0.52 & 0.10 \\
\hline 52690 & 070206.73 & -034517.4 & M1Ib comp SB & 6.58 & 2.01 & 50 & 2.36 & 0.30 \\
\hline 55057 & 071123.63 & -001806.9 & $\mathrm{~F} 2 \mathrm{~V}$ & 5.44 & 4.64 & 150 & 0.57 & 0.05 \\
\hline 55185 & 071151.86 & -002934.0 & $\mathrm{~A} 2 \mathrm{~V}$ & 4.15 & 3.90 & 155 & 0.79 & 0.05 \\
\hline 56537 & 071805.61 & +163225.7 & $\mathrm{~A} 3 \mathrm{~V} \cdots$ & 3.58 & 3.54 & 165 & 0.92 & 0.06 \\
\hline 57150 & 071818.40 & -364402.3 & $\mathrm{~B} 2 \mathrm{~V}+\cdots$ & 4.65 & 4.52 & 360 & 0.59 & 0.19 \\
\hline 56169 & 071831.98 & +492753.1 & A4IIIn & 5.00 & 4.62 & 215 & 0.57 & 0.29 \\
\hline 57167 & 071928.08 & -162341.7 & F2III/IV & 5.70 & 4.72 & 100 & 0.56 & 0.06 \\
\hline 56986 & 072007.39 & +215856.4 & FOIV $\ldots$ & 3.50 & 2.56 & 115 & 1.51 & 0.05 \\
\hline 58954 & 072707.99 & -175153.5 & F2V & 5.60 & 4.76 & 185 & 0.55 & 0.08 \\
\hline 58715 & 072709.07 & +08 1721.9 & B8Vvar & 2.89 & 3.10 & 285 & 1.11 & 0.17 \\
\hline 59037 & 072920.46 & +280706.3 & A4V & 5.07 & 4.74 & 220 & 0.53 & 0.12 \\
\hline 61715 & 073818.21 & -483605.2 & F4Iab & 5.68 & 4.11 & 55 & 0.76 & 0.06 \\
\hline 61110 & 073909.96 & +343504.7 & F3III & 4.89 & 3.84 & 90 & 0.84 & 0.07 \\
\hline
\end{tabular}


Table 4 (Continued)

\begin{tabular}{|c|c|c|c|c|c|c|c|c|}
\hline HD & $\begin{array}{l}\text { RA } \\
\text { hh:mm:ss }\end{array}$ & $\begin{array}{l}\text { DE } \\
\text { dd:mm:ss }\end{array}$ & $\begin{array}{l}\text { Spectral } \\
\text { type }\end{array}$ & $\begin{array}{l}V \\
(\mathrm{mag})\end{array}$ & $\begin{array}{l}K \\
(\mathrm{mag})\end{array}$ & $\begin{array}{l}v \sin i \\
(\mathrm{~km} / \mathrm{s})\end{array}$ & $\begin{array}{l}\theta_{\mathrm{EST}} \\
(\mathrm{mas})\end{array}$ & $\begin{array}{l}\text { Oblateness } \\
R_{b} / R_{a}-1\end{array}$ \\
\hline 61497 & 074300.46 & +584237.8 & A3IVn & 4.93 & 4.63 & 200 & 0.56 & 0.17 \\
\hline 63462 & 074805.17 & -255613.8 & B1IV:nne & 4.40 & 4.17 & 375 & 0.69 & 0.33 \\
\hline 64760 & 075318.16 & -480610.6 & B0.5Ib & 4.22 & 4.64 & 250 & 0.54 & 0.07 \\
\hline 64145 & 075329.84 & +264557.1 & A3V & 4.97 & 4.66 & 160 & 0.56 & 0.06 \\
\hline 65925 & 075928.43 & -391748.6 & F3V & 5.22 & 4.22 & 165 & 0.71 & 0.07 \\
\hline 65810 & 075952.06 & -182356.9 & A1V & 4.61 & 4.31 & 220 & 0.65 & 0.11 \\
\hline 67006 & 080827.50 & +513024.0 & $\mathrm{~A} 2 \mathrm{~V}$ & 4.78 & 4.66 & 175 & 0.55 & 0.07 \\
\hline 67797 & 080901.64 & -191442.0 & $\mathrm{~B} 5 \mathrm{~V}$ & 4.40 & 4.77 & 185 & 0.51 & 0.06 \\
\hline 72072 & 082539.14 & -710302.6 & K2III & 7.74 & 4.73 & 200 & 0.62 & 0.33 \\
\hline 71935 & 082736.65 & -530518.7 & A9/F0III/IV & 5.08 & 4.44 & 160 & 0.62 & 0.16 \\
\hline 72041 & 083130.57 & +240452.4 & F0IIIn & 5.71 & 4.91 & 110 & 0.51 & 0.10 \\
\hline 72779 & 083519.47 & +193524.3 & G0III & 6.55 & 5.00 & 85 & 0.51 & 0.05 \\
\hline 73262 & 083739.41 & +054213.7 & A1Vnn & 4.14 & 4.03 & 285 & 0.73 & 0.20 \\
\hline 75710 & 084947.65 & -451828.5 & A2III & 4.94 & 4.62 & 110 & 0.57 & 0.07 \\
\hline 76143 & 085034.68 & -664735.6 & F5IV & 5.34 & 4.32 & 140 & 0.67 & 0.10 \\
\hline 75486 & 085322.57 & +615744.0 & F2III & 5.72 & 4.88 & 115 & 0.51 & 0.11 \\
\hline 79837 & 085641.88 & -853947.6 & F0III & 5.43 & 4.67 & 115 & 0.57 & 0.11 \\
\hline 76543 & 085714.91 & +151921.8 & A5III & 5.22 & 4.87 & 90 & 0.51 & 0.06 \\
\hline 76644 & 085912.84 & +480232.5 & A7IV & 3.12 & 2.66 & 150 & 1.40 & 0.09 \\
\hline 77327 & 090337.56 & +470924.0 & A1Vn & 3.57 & 3.38 & 235 & 0.99 & 0.13 \\
\hline 77601 & 090524.11 & +483149.3 & F6II-III & 5.48 & 4.34 & 160 & 0.67 & 0.35 \\
\hline 80007 & 091312.24 & -694302.9 & A2IV & 1.67 & 1.49 & 140 & 2.38 & 0.07 \\
\hline 79439 & 091611.28 & +540118.2 & $\mathrm{A} 5 \mathrm{~V}$ & 4.80 & 4.29 & 155 & 0.66 & 0.06 \\
\hline 80081 & 091850.67 & +364810.4 & A1V & 3.82 & 3.42 & 170 & 0.99 & 0.06 \\
\hline 81471 & 092359.34 & -514413.5 & A7Iab & 6.05 & 4.27 & 65 & 0.72 & 0.05 \\
\hline 82554 & 092409.73 & -804713.9 & F3/F5IV & 5.34 & 4.20 & 160 & 0.72 & 0.09 \\
\hline 82434 & 093042.11 & -402800.8 & F2IV & 3.60 & 2.67 & 260 & 1.43 & 0.26 \\
\hline 81937 & 093131.57 & +630342.5 & FOIV & 3.65 & 2.86 & 145 & 1.30 & 0.09 \\
\hline 82621 & 093449.49 & +520305.6 & $\mathrm{~A} 2 \mathrm{~V}$ & 4.47 & 4.35 & 185 & 0.64 & 0.08 \\
\hline 83446 & 093649.66 & -492118.5 & A $5 \mathrm{~V}$ & 4.34 & 3.94 & 155 & 0.78 & 0.06 \\
\hline 83953 & 094117.03 & -233529.5 & B5V & 4.76 & 4.54 & 315 & 0.58 & 0.18 \\
\hline 85376 & 095153.02 & +242344.9 & A5IV & 5.29 & 4.66 & 130 & 0.56 & 0.07 \\
\hline 87427 & 100421.02 & -241708.1 & F0V & 5.70 & 4.87 & 180 & 0.52 & 0.07 \\
\hline 87696 & 100725.73 & +351440.9 & A7V & 4.49 & 4.00 & 160 & 0.76 & 0.06 \\
\hline 87901 & 100822.46 & +115801.9 & B7V & 1.36 & 1.64 & 330 & 2.16 & 0.23 \\
\hline 88215 & 101005.96 & -124856.4 & F2/F3IV/V & 5.30 & 4.40 & 195 & 0.65 & 0.13 \\
\hline 88824 & 101322.88 & -511358.6 & A7V & 5.27 & 4.62 & 235 & 0.58 & 0.13 \\
\hline 89080 & 101344.28 & -700216.5 & B8III & 3.29 & 3.45 & 230 & 0.94 & 0.33 \\
\hline 89254 & 101737.90 & -080408.1 & F2III & 5.25 & 4.44 & 90 & 0.63 & 0.07 \\
\hline 90132 & 102329.41 & -380035.0 & A8V & 5.34 & 4.69 & 270 & 0.56 & 0.18 \\
\hline 91465 & 103201.48 & -614107.3 & B4Vne & 3.30 & 3.24 & 305 & 1.05 & 0.15 \\
\hline
\end{tabular}


Table 4 (Continued)

\begin{tabular}{|c|c|c|c|c|c|c|c|c|}
\hline HD & $\begin{array}{l}\text { RA } \\
\text { hh:mm:ss }\end{array}$ & $\begin{array}{l}\text { DE } \\
\text { dd:mm:ss }\end{array}$ & $\begin{array}{l}\text { Spectral } \\
\text { type }\end{array}$ & $\begin{array}{l}V \\
(\mathrm{mag})\end{array}$ & $\begin{array}{l}K \\
\text { (mag) }\end{array}$ & $\begin{array}{l}v \sin i \\
(\mathrm{~km} / \mathrm{s})\end{array}$ & $\begin{array}{l}\theta_{\mathrm{EST}} \\
(\mathrm{mas})\end{array}$ & $\begin{array}{l}\text { Oblateness } \\
R_{b} / R_{a}-1\end{array}$ \\
\hline 91312 & 103314.00 & +402531.9 & A7IV & 4.72 & 4.20 & 135 & 0.69 & 0.08 \\
\hline 94601 & 105536.85 & +244459.1 & A1 & 4.30 & 4.35 & 185 & 0.63 & 0.08 \\
\hline 95370 & 110009.25 & -421333.1 & A3IV & 4.37 & 4.07 & 115 & 0.73 & 0.05 \\
\hline 96202 & 110520.03 & -271736.9 & F3IV/V & 4.92 & 4.09 & 240 & 0.74 & 0.22 \\
\hline 97603 & 111406.41 & +203126.5 & $\mathrm{~A} 4 \mathrm{~V}$ & 2.56 & 2.14 & 195 & 1.78 & 0.09 \\
\hline 98058 & 111639.76 & -033905.5 & A7IVn & 4.45 & 4.13 & 240 & 0.71 & 0.29 \\
\hline 98718 & 112100.44 & -542927.7 & B5Vn & 3.90 & 4.31 & 340 & 0.63 & 0.22 \\
\hline 100841 & 113546.93 & -630111.4 & B9II: & 3.11 & 3.07 & 165 & 1.14 & 0.17 \\
\hline 100889 & 113640.95 & -094808.1 & B9.5Vn & 4.70 & 4.78 & 190 & 0.51 & 0.07 \\
\hline 101431 & 114012.82 & -344440.8 & B9V & 4.70 & 4.79 & 245 & 0.51 & 0.13 \\
\hline 102124 & 114517.00 & +08 1529.4 & $\mathrm{~A} 4 \mathrm{~V}$ & 4.84 & 4.41 & 170 & 0.63 & 0.07 \\
\hline 102776 & 114941.09 & -634718.6 & B3V & 4.30 & 4.68 & 270 & 0.53 & 0.11 \\
\hline 103287 & 115349.74 & +534141.0 & A0V SB & 2.41 & 2.43 & 170 & 1.52 & 0.06 \\
\hline 106490 & 121508.76 & -584456.0 & B2IV & 2.79 & 3.53 & 175 & 0.88 & 0.06 \\
\hline 106591 & 121525.45 & +570157.4 & A3Vvar & 3.32 & 3.10 & 180 & 1.13 & 0.07 \\
\hline 106661 & 121600.23 & +145356.9 & A3V & 5.09 & 4.89 & 175 & 0.50 & 0.07 \\
\hline 106911 & 121820.94 & -791844.2 & $\mathrm{~B} 5 \mathrm{Vn}$ & 4.24 & 4.56 & 260 & 0.56 & 0.12 \\
\hline 108283 & 122624.07 & +271605.7 & F0p & 4.92 & 4.15 & 230 & 0.72 & 0.12 \\
\hline 108483 & 122802.41 & -501350.2 & B3V & 3.91 & 4.48 & 215 & 0.58 & 0.07 \\
\hline 108722 & 122927.05 & +240632.1 & F5III & 5.47 & 4.55 & 90 & 0.60 & 0.07 \\
\hline 108767 & 122951.98 & -163054.3 & B9.5V & 2.94 & 3.00 & 160 & 1.17 & 0.05 \\
\hline 109026 & 123228.11 & -720758.7 & $\mathrm{~B} 5 \mathrm{~V}$ & 3.84 & 4.25 & 205 & 0.64 & 0.07 \\
\hline 109141 & 123236.09 & -135132.3 & F3IV/V & 5.74 & 4.88 & 135 & 0.52 & 0.06 \\
\hline 109387 & 123329.04 & +694717.6 & B6IIIp & 3.85 & 3.82 & 230 & 0.80 & 0.29 \\
\hline 109536 & 123545.61 & -410119.0 & A7III & 5.12 & 4.57 & 110 & 0.59 & 0.09 \\
\hline 109787 & 123742.33 & -483228.6 & $\mathrm{~A} 2 \mathrm{~V}$ & 3.85 & 3.71 & 330 & 0.85 & 0.30 \\
\hline 110411 & 124153.01 & +101409.0 & $\mathrm{A} 0 \mathrm{~V}$ & 4.88 & 4.68 & 175 & 0.55 & 0.07 \\
\hline 110335 & 124156.60 & -594108.9 & B6IV & 4.91 & 4.79 & 240 & 0.52 & 0.18 \\
\hline 110432 & 124250.28 & -630331.0 & B2pe & 5.27 & 4.04 & 275 & 0.78 & 0.10 \\
\hline 112429 & 125528.56 & +652618.8 & A5n & 5.23 & 4.43 & 160 & 0.63 & 0.06 \\
\hline 113314 & 130333.35 & -493138.1 & $\mathrm{~A} 0 \mathrm{~V}$ & 4.83 & 4.74 & 240 & 0.53 & 0.13 \\
\hline 114529 & 131217.63 & -595513.9 & B8V & 4.58 & 4.71 & 280 & 0.53 & 0.17 \\
\hline 116842 & 132513.42 & +54 5916.8 & A5V SB & 3.99 & 3.15 & 230 & 1.15 & 0.13 \\
\hline 117150 & 132925.26 & -510954.4 & $\mathrm{~A} 0 \mathrm{~V}$ & 5.04 & 4.72 & 315 & 0.54 & 0.25 \\
\hline 118232 & 133427.37 & +490057.3 & A5V & 4.68 & 4.27 & 175 & 0.67 & 0.07 \\
\hline 118098 & 133441.75 & -003545.4 & A3V & 3.38 & 3.22 & 190 & 1.07 & 0.08 \\
\hline 118261 & 133712.20 & -614129.7 & F6V & 5.63 & 4.44 & 150 & 0.64 & 0.06 \\
\hline 118623 & 133727.70 & +361741.4 & A7III & 4.82 & 4.15 & 235 & 0.72 & 0.27 \\
\hline 118716 & 133953.27 & -532758.9 & B1III & 2.29 & 3.04 & 140 & 1.11 & 0.05 \\
\hline 120315 & 134732.55 & +491847.9 & B3V SB & 1.85 & 2.27 & 195 & 1.61 & 0.05 \\
\hline 120324 & 134937.01 & -422825.3 & B2IV-Ve & 3.47 & 4.01 & 180 & 0.72 & 0.05 \\
\hline
\end{tabular}


Table 4 (Continued)

\begin{tabular}{|c|c|c|c|c|c|c|c|c|}
\hline HD & $\begin{array}{l}\text { RA } \\
\text { hh:mm:ss }\end{array}$ & $\begin{array}{l}\text { DE } \\
\text { dd:mm:ss }\end{array}$ & $\begin{array}{l}\text { Spectral } \\
\text { type }\end{array}$ & $\begin{array}{l}V \\
\text { (mag) }\end{array}$ & $\begin{array}{l}K \\
\text { (mag) }\end{array}$ & $\begin{array}{l}v \sin i \\
(\mathrm{~km} / \mathrm{s})\end{array}$ & $\begin{array}{l}\theta_{\mathrm{EST}} \\
(\mathrm{mas})\end{array}$ & $\begin{array}{l}\text { Oblateness } \\
R_{b} / R_{a}-1\end{array}$ \\
\hline 121263 & 135532.43 & -471717.8 & B2.5IV & 2.55 & 3.22 & 225 & 1.02 & 0.11 \\
\hline 122408 & 140138.78 & +013240.5 & A3V & 4.23 & 4.09 & 165 & 0.71 & 0.06 \\
\hline 123255 & 140642.91 & -091848.7 & F2IV & 5.46 & 4.60 & 140 & 0.59 & 0.09 \\
\hline 124675 & 141328.95 & +514724.0 & A8IV & 4.53 & 4.10 & 130 & 0.72 & 0.07 \\
\hline 124367 & 141457.16 & -570509.9 & B4Vne & 5.03 & 4.77 & 270 & 0.53 & 0.12 \\
\hline 125238 & 141924.23 & -460329.1 & B2.5IV & 3.55 & 4.10 & 235 & 0.69 & 0.12 \\
\hline 126248 & 142411.39 & +054912.4 & A5V & 5.10 & 4.77 & 190 & 0.53 & 0.09 \\
\hline 127762 & 143204.76 & +38 1828.4 & A7IIIvar & 3.04 & 2.51 & 145 & 1.51 & 0.17 \\
\hline 127972 & 143530.45 & -420927.9 & $\mathrm{~B} 1 \mathrm{Vn}+\mathrm{A}$ & 2.33 & 2.75 & 345 & 1.29 & 0.16 \\
\hline 128345 & 143753.25 & -492532.7 & $\mathrm{~B} 5 \mathrm{~V}$ & 4.05 & 4.51 & 185 & 0.57 & 0.06 \\
\hline 129246 & 144108.92 & +134342.0 & A3IVn & 3.78 & 3.70 & 150 & 0.85 & 0.09 \\
\hline 129988 & 144459.25 & +270427.0 & A0 & 2.35 & 0.12 & 165 & 4.96 & 0.06 \\
\hline 129422 & 144517.25 & -625231.6 & A7Vn & 5.36 & 4.58 & 270 & 0.59 & 0.19 \\
\hline 129926 & 144600.18 & -252634.5 & $\mathrm{~F} 0 \mathrm{~V}+\mathrm{G} / \mathrm{K}$ & 5.15 & 4.43 & 210 & 0.63 & 0.10 \\
\hline 130109 & 144614.99 & +015334.6 & A0V & 3.73 & 3.65 & 340 & 0.87 & 0.31 \\
\hline 131492 & 145644.00 & -624651.6 & B4Vnp & 5.08 & 4.60 & 210 & 0.58 & 0.07 \\
\hline 134481 & 151156.16 & -484415.7 & B9V & 3.88 & 3.97 & 205 & 0.75 & 0.09 \\
\hline 135742 & 151700.47 & -092258.3 & $\mathrm{~B} 8 \mathrm{~V}$ & 2.61 & 2.91 & 230 & 1.21 & 0.11 \\
\hline 135734 & 151832.05 & -475230.7 & $\mathrm{~B} 8 \mathrm{~V}$ & 4.27 & 4.43 & 400 & 0.60 & 0.41 \\
\hline 135382 & 151854.69 & -684046.1 & A1V & 2.87 & 2.53 & 225 & 1.48 & 0.12 \\
\hline 137422 & 152043.75 & +715002.3 & A3II-III & 3.00 & 2.71 & 160 & 1.36 & 0.20 \\
\hline 136298 & 152122.34 & -403850.9 & B1.5IV & 3.22 & 3.96 & 225 & 0.72 & 0.10 \\
\hline 137058 & 152520.25 & -384400.9 & A0V & 4.60 & 4.48 & 300 & 0.60 & 0.22 \\
\hline 137898 & 152838.29 & +015031.8 & A8IV & 5.15 & 4.59 & 130 & 0.58 & 0.07 \\
\hline 138629 & 153146.99 & +405357.7 & $\mathrm{~A} 5 \mathrm{~V}$ & 4.98 & 4.61 & 175 & 0.57 & 0.07 \\
\hline 138749 & 153255.80 & +312133.0 & B6Vnn & 4.14 & 4.43 & 385 & 0.60 & 0.32 \\
\hline 138690 & 153508.46 & -411000.1 & B2IV & 2.80 & 3.38 & 270 & 0.96 & 0.16 \\
\hline 140159 & 154133.09 & +194013.8 & A1V & 4.51 & 4.31 & 175 & 0.65 & 0.07 \\
\hline 142105 & 154403.46 & +774740.2 & $\mathrm{~A} 3 \mathrm{Vn}$ & 4.29 & 4.20 & 210 & 0.68 & 0.10 \\
\hline 140417 & 154404.42 & -154021.6 & A6IV & 5.41 & 4.82 & 115 & 0.52 & 0.05 \\
\hline 141003 & 154611.21 & +152518.9 & A3V & 3.65 & 3.55 & 185 & 0.92 & 0.08 \\
\hline 141637 & 155058.75 & -254504.4 & $\mathrm{~B} 1.5 \mathrm{Vn}$ & 4.63 & 4.78 & 310 & 0.51 & 0.13 \\
\hline 141851 & 155115.65 & -030525.5 & A3Vn & 5.09 & 4.70 & 185 & 0.55 & 0.08 \\
\hline 142114 & 155336.73 & -251937.5 & $\mathrm{~B} 2.5 \mathrm{Vn}$ & 4.59 & 4.79 & 320 & 0.51 & 0.15 \\
\hline 141891 & 155508.81 & -632547.1 & F2III & 2.83 & 2.15 & 90 & 1.80 & 0.07 \\
\hline 143466 & 155747.59 & +544458.2 & FOIV & 4.96 & 4.28 & 140 & 0.68 & 0.08 \\
\hline 142983 & 155811.38 & -141645.5 & B8Ia/Iab & 4.95 & 4.59 & 405 & 0.57 & 0.43 \\
\hline 143118 & 160007.34 & -382347.9 & B2.5IV & 3.42 & 4.09 & 240 & 0.68 & 0.12 \\
\hline 143275 & 160020.01 & -223717.8 & B0.2IV & 2.29 & 2.43 & 180 & 1.52 & 0.05 \\
\hline 143474 & 160332.22 & -574629.5 & A7IV & 4.63 & 4.10 & 175 & 0.73 & 0.13 \\
\hline 144294 & 160635.56 & -364808.0 & $\mathrm{~B} 2.5 \mathrm{Vn}$ & 4.22 & 4.70 & 320 & 0.52 & 0.15 \\
\hline
\end{tabular}


Table 4 (Continued)

\begin{tabular}{|c|c|c|c|c|c|c|c|c|}
\hline HD & $\begin{array}{l}\text { RA } \\
\text { hh:mm:ss }\end{array}$ & $\begin{array}{l}\text { DE } \\
\text { dd:mm:ss }\end{array}$ & $\begin{array}{l}\text { Spectral } \\
\text { type }\end{array}$ & $\begin{array}{l}V \\
(\mathrm{mag})\end{array}$ & $\begin{array}{l}K \\
(\mathrm{mag})\end{array}$ & $\begin{array}{l}v \sin i \\
(\mathrm{~km} / \mathrm{s})\end{array}$ & $\begin{array}{l}\theta_{\mathrm{EST}} \\
(\mathrm{mas})\end{array}$ & $\begin{array}{l}\text { Oblateness } \\
R_{b} / R_{a}-1\end{array}$ \\
\hline 144668 & 160834.29 & -390618.1 & A8/A9 & 7.00 & 4.39 & 180 & 0.71 & 0.07 \\
\hline 145502 & 161159.74 & -192738.3 & B2IV & 4.00 & 3.88 & 210 & 0.79 & 0.09 \\
\hline 147547 & 162155.24 & +190910.9 & A9III & 3.74 & 2.94 & 145 & 1.25 & 0.18 \\
\hline 147933 & 162535.12 & -232649.6 & $\mathrm{~B} 2 \mathrm{~V}$ & 4.57 & 3.17 & 300 & 1.17 & 0.13 \\
\hline 149630 & 163406.19 & +422612.8 & B9Vvar & 4.20 & 4.05 & 280 & 0.73 & 0.18 \\
\hline 149757 & 163709.53 & -103401.7 & $09.5 \mathrm{~V}$ & 2.54 & 2.68 & 385 & 1.35 & 0.20 \\
\hline 151890 & 165152.24 & -380250.4 & $\mathrm{~B} 1.5 \mathrm{IV}+\mathrm{B}$ & 3.00 & 3.70 & 235 & 0.82 & 0.11 \\
\hline 152427 & 165401.02 & -184436.1 & K1III & 8.65 & 5.16 & 92 & 0.52 & 0.11 \\
\hline 154494 & 170522.66 & +124427.1 & A4IV & 4.89 & 4.61 & 115 & 0.57 & 0.05 \\
\hline 155203 & 171209.18 & -431418.6 & F3p & 3.32 & 2.26 & 150 & 1.75 & 0.05 \\
\hline 156164 & 171501.92 & +245022.5 & A3IVv SB & 3.12 & 2.81 & 295 & 1.30 & 0.32 \\
\hline 156729 & 171740.29 & +371728.8 & $\mathrm{~A} 2 \mathrm{~V}$ & 4.64 & 4.44 & 155 & 0.61 & 0.05 \\
\hline 157778 & 172340.97 & +370845.3 & B9.5III & 4.15 & 4.23 & 195 & 0.66 & 0.23 \\
\hline 157246 & 172523.66 & -562239.7 & $\mathrm{~B} 1 \mathrm{Ib}$ & 3.31 & 3.79 & 285 & 0.79 & 0.12 \\
\hline 158352 & 172849.69 & +001950.1 & A8V & 5.41 & 4.81 & 190 & 0.53 & 0.08 \\
\hline 158094 & 173105.98 & -604101.0 & B8V & 3.60 & 3.71 & 255 & 0.84 & 0.13 \\
\hline 158643 & 173124.95 & -235745.3 & $\mathrm{~A} 0 \mathrm{~V}$ & 4.78 & 4.30 & 220 & 0.66 & 0.11 \\
\hline 158427 & 173150.52 & -495233.5 & B2Vne & 2.84 & 2.49 & 315 & 1.51 & 0.14 \\
\hline 166205 & 173212.90 & +863510.8 & A1Vn & 4.35 & 4.26 & 180 & 0.66 & 0.07 \\
\hline 159561 & 173456.00 & +123338.1 & A5III & 2.08 & 1.68 & 230 & 2.20 & 0.25 \\
\hline 159532 & 173719.13 & -425952.2 & F1II & 1.86 & 0.84 & 125 & 3.34 & 0.19 \\
\hline 159975 & 173750.72 & -080707.4 & B8II-IIIMNp & 4.58 & 4.25 & 135 & 0.67 & 0.09 \\
\hline 160365 & 173857.87 & +131945.0 & F6III & 6.12 & 4.70 & 90 & 0.58 & 0.07 \\
\hline 161868 & 174753.57 & +024226.9 & $\mathrm{~A} 0 \mathrm{~V}$ & 3.75 & 3.62 & 220 & 0.89 & 0.11 \\
\hline 163955 & 175947.56 & -234857.6 & B9V & 4.74 & 4.47 & 250 & 0.60 & 0.14 \\
\hline 164577 & 180145.19 & +011818.4 & $\mathrm{~A} 2 \mathrm{Vn}$ & 4.42 & 4.23 & 260 & 0.67 & 0.16 \\
\hline 166045 & 180749.56 & +260604.4 & $\mathrm{~A} 3 \mathrm{~V}$ & 5.83 & 5.12 & 175 & 0.46 & 0.07 \\
\hline 168914 & 182101.02 & +285211.4 & A7V & 5.12 & 4.48 & 195 & 0.61 & 0.09 \\
\hline 170073 & 182354.65 & +584802.1 & A3V & 4.98 & 4.78 & 180 & 0.52 & 0.07 \\
\hline 169022 & 182410.35 & -342303.5 & B9.5III & 1.79 & 1.77 & 175 & 2.07 & 0.18 \\
\hline 169702 & 182413.80 & +393026.1 & A3IVn & 5.11 & 4.86 & 200 & 0.51 & 0.17 \\
\hline 169985 & 182712.51 & +001146.1 & $\mathrm{GOIII}+\cdots$ & 5.20 & 3.39 & 270 & 1.08 & 0.22 \\
\hline 170296 & 182911.85 & -143356.9 & A1IV/V & 4.67 & 4.36 & 255 & 0.64 & 0.21 \\
\hline 170479 & 183104.85 & -325920.4 & A5V & 5.37 & 4.87 & 160 & 0.51 & 0.06 \\
\hline 169978 & 183122.43 & -621641.5 & B8III & 4.63 & 4.83 & 125 & 0.50 & 0.08 \\
\hline 172365 & 183936.88 & +051551.4 & F8Ib-II & 6.36 & 4.46 & 67.3 & 0.66 & 0.09 \\
\hline 172777 & 184346.94 & -381923.9 & A0/A1V & 5.11 & 4.86 & 175 & 0.50 & 0.07 \\
\hline 173582 & 184420.34 & +394011.9 & F1V & 4.67 & 4.23 & 195 & 0.68 & 0.09 \\
\hline 173607 & 184422.78 & +393645.3 & $\mathrm{A} 8 \mathrm{Vn}$ & 4.59 & 4.16 & 175 & 0.70 & 0.07 \\
\hline 173649 & 184448.19 & +373540.4 & F0IVvar & 5.73 & 4.96 & 240 & 0.50 & 0.29 \\
\hline 172555 & 184526.86 & -645215.2 & A7V & 4.78 & 4.30 & 175 & 0.66 & 0.07 \\
\hline
\end{tabular}


Table 4 (Continued)

\begin{tabular}{|c|c|c|c|c|c|c|c|c|}
\hline HD & $\begin{array}{l}\text { RA } \\
\text { hh:mm:ss }\end{array}$ & $\begin{array}{l}\text { DE } \\
\text { dd:mm:ss }\end{array}$ & $\begin{array}{l}\text { Spectral } \\
\text { type }\end{array}$ & $\begin{array}{l}V \\
\text { (mag) }\end{array}$ & $\begin{array}{l}K \\
\text { (mag) }\end{array}$ & $\begin{array}{l}v \sin i \\
(\mathrm{~km} / \mathrm{s})\end{array}$ & $\begin{array}{l}\theta_{\mathrm{EST}} \\
(\mathrm{mas})\end{array}$ & $\begin{array}{l}\text { Oblateness } \\
R_{b} / R_{a}-1\end{array}$ \\
\hline 174602 & 184952.92 & +323303.9 & A3V & 5.22 & 4.90 & 150 & 0.50 & 0.05 \\
\hline 173948 & 185213.04 & -621115.2 & B2II-III & 4.22 & 4.39 & 190 & 0.61 & 0.10 \\
\hline 175824 & 185447.17 & +485135.0 & F3III & 5.84 & 4.67 & 80 & 0.58 & 0.05 \\
\hline 175191 & 185515.92 & -261747.7 & $\mathrm{~B} 2.5 \mathrm{~V}$ & 2.05 & 2.44 & 205 & 1.49 & 0.06 \\
\hline 175639 & 185614.61 & +041207.4 & $\mathrm{~A} 5 \mathrm{Vn}$ & 4.98 & 4.46 & 200 & 0.62 & 0.10 \\
\hline 175813 & 185843.47 & -370625.5 & F3IV/V & 4.83 & 3.88 & 175 & 0.82 & 0.11 \\
\hline 176723 & 190317.69 & -381511.5 & F2III/IV & 5.73 & 4.87 & 265 & 0.52 & 0.27 \\
\hline 177724 & 190524.61 & +135149.4 & $\mathrm{~A} 0 \mathrm{Vn}$ & 2.99 & 2.88 & 345 & 1.25 & 0.32 \\
\hline 177756 & 190614.95 & -045256.4 & B9Vn & 3.43 & 3.56 & 160 & 0.90 & 0.05 \\
\hline 178233 & 190637.68 & +283742.2 & F0III & 5.53 & 4.81 & 165 & 0.53 & 0.24 \\
\hline 178449 & 190725.50 & +323006.0 & F0V & 5.20 & 4.19 & 155 & 0.71 & 0.05 \\
\hline 178596 & 190859.92 & +060424.2 & FOIII-IV & 5.23 & 4.33 & 125 & 0.66 & 0.09 \\
\hline 178253 & 190928.28 & -375415.3 & A0/A1V & 4.11 & 4.05 & 225 & 0.73 & 0.11 \\
\hline 180868 & 191749.00 & +113543.4 & FOIV & 5.28 & 4.71 & 115 & 0.55 & 0.05 \\
\hline 181623 & 192313.06 & -444758.7 & F2III & 4.27 & 2.93 & 155 & 1.30 & 0.22 \\
\hline 184006 & 192942.34 & +514346.1 & $\mathrm{~A} 5 \mathrm{Vn}$ & 3.76 & 3.60 & 220 & 0.90 & 0.12 \\
\hline 186005 & 194231.09 & -160726.3 & F3IV/V & 5.06 & 4.28 & 160 & 0.68 & 0.09 \\
\hline 186882 & 194458.44 & +450750.5 & B9.5III & 2.86 & 2.68 & 140 & 1.37 & 0.11 \\
\hline 187076 & 194723.27 & +183203.3 & $\mathrm{M} 2 \mathrm{II}+\mathrm{B} 6$ & 3.68 & -0.56 & 50 & 7.56 & 0.07 \\
\hline 187362 & 194858.65 & +190831.1 & $\mathrm{A} 3 \mathrm{~V}$ & 5.01 & 4.73 & 240 & 0.54 & 0.14 \\
\hline 186219 & 194925.29 & -723012.3 & A4III & 5.39 & 4.80 & 125 & 0.53 & 0.11 \\
\hline 187642 & 195046.68 & +085202.6 & A7IV-V & 0.76 & 0.10 & 240 & 4.61 & 0.20 \\
\hline 189037 & 195537.82 & +522620.5 & $\mathrm{~A} 4 \mathrm{Vn}$ & 4.91 & 4.49 & 285 & 0.60 & 0.21 \\
\hline 190004 & 200201.37 & +245616.3 & F2III & 5.23 & 4.30 & 150 & 0.68 & 0.21 \\
\hline 192514 & 201318.04 & +464856.4 & A5IIIn & 4.80 & 4.41 & 145 & 0.63 & 0.17 \\
\hline 192696 & 201323.80 & +563403.1 & A3IV-Vn & 4.28 & 4.08 & 280 & 0.72 & 0.28 \\
\hline 192518 & 201414.52 & +284141.5 & A7IVn & 5.19 & 4.65 & 190 & 0.57 & 0.16 \\
\hline 192425 & 201416.59 & +151150.9 & $\mathrm{~A} 2 \mathrm{~V}$ & 4.94 & 4.77 & 160 & 0.52 & 0.06 \\
\hline 195627 & 203534.77 & -603452.7 & F1III & 4.75 & 4.04 & 150 & 0.75 & 0.20 \\
\hline 197157 & 204402.19 & -515515.0 & A6:var & 4.51 & 3.82 & 150 & 0.83 & 0.05 \\
\hline 197937 & 204829.00 & -435917.8 & F1IV & 5.11 & 4.20 & 115 & 0.71 & 0.06 \\
\hline 199611 & 205625.44 & +504343.1 & FOIII & 5.83 & 4.92 & 125 & 0.51 & 0.13 \\
\hline 199629 & 205710.41 & +411001.9 & A1Vn & 3.94 & 3.52 & 250 & 0.94 & 0.15 \\
\hline 200120 & 205949.55 & +473115.4 & Blne & 4.74 & 4.35 & 379 & 0.64 & 0.20 \\
\hline 199532 & 210443.03 & -770122.3 & F4III & 5.13 & 3.60 & 85 & 0.96 & 0.06 \\
\hline 177482 & 210846.01 & -885723.4 & FOIII & 5.45 & 4.67 & 145 & 0.56 & 0.18 \\
\hline 202904 & 211755.07 & +345348.8 & B2Vne & 4.41 & 4.48 & 255 & 0.59 & 0.09 \\
\hline 203280 & 211834.58 & +623507.6 & A7IV-V & 2.45 & 2.07 & 245 & 1.84 & 0.21 \\
\hline 202730 & 211951.88 & -532657.4 & $\mathrm{~A} 5 \mathrm{~V}$ & 4.39 & 4.15 & 210 & 0.70 & 0.11 \\
\hline 203803 & 212358.74 & +241626.7 & F1IV & 5.70 & 4.92 & 110 & 0.51 & 0.05 \\
\hline 205114 & 213127.46 & +523711.5 & $\mathrm{G} 2 \mathrm{Ib}+\cdots$ & 6.17 & 3.87 & 50 & 0.89 & 0.07 \\
\hline
\end{tabular}


Table 4 (Continued)

\begin{tabular}{|c|c|c|c|c|c|c|c|c|}
\hline $\mathrm{HD}$ & $\begin{array}{l}\text { RA } \\
\text { hh:mm:ss }\end{array}$ & $\begin{array}{l}\text { DE } \\
\text { dd:mm:ss }\end{array}$ & $\begin{array}{l}\text { Spectral } \\
\text { type }\end{array}$ & $\begin{array}{l}V \\
(\mathrm{mag})\end{array}$ & $\begin{array}{l}K \\
(\mathrm{mag})\end{array}$ & $\begin{array}{l}v \sin i \\
(\mathrm{~km} / \mathrm{s})\end{array}$ & $\begin{array}{l}\theta_{\mathrm{EST}} \\
(\mathrm{mas})\end{array}$ & $\begin{array}{l}\text { Oblateness } \\
R_{b} / R_{a}-1\end{array}$ \\
\hline 205835 & 213656.98 & +402448.6 & A5V & 5.04 & 4.51 & 180 & 0.60 & 0.08 \\
\hline 205637 & 213704.82 & -192757.6 & B3V:p & 4.51 & 4.79 & 225 & 0.51 & 0.07 \\
\hline 205767 & 213745.04 & -075114.9 & A7V & 4.68 & 4.25 & 165 & 0.67 & 0.06 \\
\hline 205852 & 213745.37 & +191906.9 & F1IV & 5.46 & 4.61 & 180 & 0.58 & 0.15 \\
\hline 207155 & 214744.17 & -305353.9 & A1V & 5.02 & 4.85 & 175 & 0.50 & 0.07 \\
\hline 208450 & 215755.03 & -545933.2 & FOIV & 4.40 & 3.42 & 130 & 1.02 & 0.07 \\
\hline 209409 & 220318.83 & -020919.2 & B7IVe & 4.74 & 4.66 & 305 & 0.55 & 0.35 \\
\hline 209952 & 220813.88 & -465738.2 & B7IV & 1.73 & 2.02 & 250 & 1.82 & 0.21 \\
\hline 210049 & 220822.95 & -325918.2 & $\mathrm{~A} 2 \mathrm{~V}$ & 4.50 & 4.31 & 300 & 0.65 & 0.23 \\
\hline 210334 & 220840.86 & +454431.7 & K2III comp & 6.11 & 4.27 & 70 & 0.72 & 0.07 \\
\hline 210271 & 220955.71 & -340054.1 & A5IV & 5.37 & 4.75 & 260 & 0.54 & 0.35 \\
\hline 210459 & 220959.25 & +331041.8 & F5III & 4.28 & 3.12 & 135 & 1.18 & 0.17 \\
\hline 210839 & 221130.58 & +592452.3 & O6e & 5.05 & 4.50 & 275 & 0.60 & 0.10 \\
\hline 212581 & 222719.87 & -645759.0 & B8V & 4.51 & 4.55 & 240 & 0.57 & 0.12 \\
\hline 213310 & 222931.82 & +474224.8 & M0II & 4.34 & 0.27 & 50 & 5.13 & 0.10 \\
\hline 213998 & 223521.33 & -000702.5 & B9IV-Vn & 4.04 & 4.24 & 280 & 0.66 & 0.24 \\
\hline 214748 & 224039.33 & -270237.0 & B8V & 4.18 & 4.40 & 375 & 0.61 & 0.34 \\
\hline 214923 & 224127.67 & +104953.0 & $\mathrm{~B} 8.5 \mathrm{~V}$ & 3.41 & 3.57 & 185 & 0.90 & 0.07 \\
\hline 215789 & 224833.20 & -511900.1 & $\mathrm{~A} 3 \mathrm{~V}$ & 3.49 & 3.19 & 270 & 1.09 & 0.18 \\
\hline 217675 & 230155.25 & +421933.5 & B6pv SB & 3.62 & 3.89 & 320 & 0.77 & 0.20 \\
\hline 217782 & 230236.34 & +424528.1 & $\mathrm{~A} 3 \mathrm{Vn}$ & 5.09 & 4.69 & 205 & 0.55 & 0.10 \\
\hline 218045 & 230445.62 & +151219.3 & B9.5III & 2.49 & 2.65 & 150 & 1.37 & 0.12 \\
\hline 217831 & 230452.15 & -684913.4 & F4III & 5.53 & 4.69 & 120 & 0.56 & 0.13 \\
\hline 218918 & 231144.19 & +084312.5 & $\mathrm{~A} 5 \mathrm{Vn}$ & 5.15 & 4.74 & 220 & 0.54 & 0.12 \\
\hline 219586 & 231537.71 & +7053 17.1 & FoIV & 5.55 & 4.81 & 150 & 0.53 & 0.10 \\
\hline 219571 & 231725.81 & -581409.3 & F1III & 3.99 & 3.04 & 95 & 1.21 & 0.07 \\
\hline 219688 & 231754.20 & -091057.0 & B5Vn & 4.41 & 4.76 & 340 & 0.51 & 0.22 \\
\hline 220061 & 232038.22 & +234425.3 & $\mathrm{~A} 5 \mathrm{~V}$ & 4.58 & 4.09 & 150 & 0.73 & 0.05 \\
\hline 221565 & 233316.63 & -205452.3 & A0V & 4.70 & 4.52 & 385 & 0.59 & 0.43 \\
\hline 222095 & 233750.94 & -452932.4 & $\mathrm{~A} 2 \mathrm{~V}$ & 4.74 & 4.52 & 165 & 0.59 & 0.06 \\
\hline 222439 & 234024.44 & +442002.3 & B9IVn & 4.15 & 4.57 & 190 & 0.56 & 0.12 \\
\hline 223352 & 234855.48 & -280748.1 & $\mathrm{~A} 0 \mathrm{~V}$ & 4.59 & 4.53 & 280 & 0.58 & 0.19 \\
\hline 223781 & 235237.12 & +105650.4 & $\mathrm{~A} 4 \mathrm{Vn}$ & 5.30 & 4.71 & 170 & 0.55 & 0.07 \\
\hline 224392 & 235734.97 & -641753.1 & A1V & 5.00 & 4.82 & 250 & 0.51 & 0.15 \\
\hline 224686 & 235954.91 & -653437.5 & B9IV & 4.49 & 4.60 & 295 & 0.56 & 0.36 \\
\hline
\end{tabular}

\section{References}

Abney WDW (1877) Mon Not R Astron Soc 37:278

Absil O, di Folco E, Mérand A, Augereau J-C, Coudé Du Foresto V, Aufdenberg JP, Kervella P, Ridgway ST, Berger DH, Ten Brummelaar TA, Sturmann J, Sturmann L, Turner NH, McAlister HA (2006) Astron Astrophys 452:237 
Abt HA, Morrell NI (1995) Astrophys J Suppl Ser 99:135

Abt HA, Moyd KI (1973) Astrophys J 182:809

Aerts C, Puls J, Godart M, Dupret M-A (2009) Astron Astrophys 508:409

Akeson RL, Ciardi DR, Millan-Gabet R, Merand A, Folco ED, Monnier JD, Beichman CA, Absil O, Aufdenberg J, McAlister H, Brummelaar TT, Sturmann J, Sturmann L, Turner N (2009) Astrophys J 691:1896

Armstrong JT, Mozurkewich D, Rickard LJ, Hutter DJ, Benson JA, Bowers PF, Elias NM II, Hummel CA, Johnston KJ, Buscher DF, Clark JH III, Ha L, Ling L-C, White NM, Simon RS (1998) Astrophys J 496:550

Asplund M, Grevesse N, Sauval AJ, Scott P (2009) Annu Rev Astron Astrophys 47:481

Aufdenberg JP, Mérand A, Coudé du Foresto V, Absil O, Di Folco E, Kervella P, Ridgway ST, Berger DH, ten Brummelaar TA, McAlister HA, Sturmann J, Sturmann L, Turner NH (2006) Astrophys J 645:664

Aufdenberg JP, Ireland MJ, Mérand A, Coudé Du Foresto V, Absil O, Folco ED, Kervella P, Bagnuolo WG, Gies DR, Ridgway ST, Berger DH, Ten Brummelaar TA, McAlister HA, Sturmann J, Sturmann L, Turner NH, Jacob AP (2007) In: Hartkopf WI, Guinan EF, Harmanec P (eds) IAU symposium, vol 240, pp 271-280

Aumann HH, Beichman CA, Gillett FC, de Jong T, Houck JR, Low FJ, Neugebauer G, Walker RG, Wesselius PR (1984) Astrophys J Lett 278:L23

Barnes JW (2009) Astrophys J 705:683

Baron F, Monnier JD, Kloppenborg B (2010) In: Society of Photo-Optical Instrumentation Engineers (SPIE) conference series, vol 7734

Baron RL, French RG, Elliot JL (1989) Icarus 78:119

Berger J-P, Zins G, Lazareff B, Lebouquin J-B, Jocou L, Kern P, Millan-Gabet R, Traub W, Haguenauer P, Absil O, Augereau J-C, Benisty M, Blind N, Bonfils X, Delboulbe A, Feautrier P, Germain M, Gillier D, Gitton P, Kiekebusch M, Knudstrup J, Lizon J-L, Magnard Y, Malbet F, Maurel D, Menard F, Micallef M, Michaud L, Morel S, Moulin T, Popovic D, Perraut K, Rabou P, Rochat S, Roussel F, Roux A, Stadler E, Tatulli E (2010) In: Society of Photo-Optical Instrumentation Engineers (SPIE) conference series, vol 7734

Bernacca PL, Perinotto M (1970) Contrib Oss Astrofis Univ Padova Asiago 239:1

Boden AF, Koresko CD, van Belle GT, Colavita MM, Dumont PJ, Gubler J, Kulkarni SR, Lane BF, Mobley D, Shao M, Wallace JK, The PTI Collaboration, Henry GW (1999) Astrophys J 515:356

Born M, Wolf E (eds) (1980) Principles of optics electromagnetic theory of propagation, interference and diffraction of light

Bracewell RN (ed) (2000) The Fourier transform and its applications

Brunet $\mathrm{P}$ (1931) L'introduction des théories de Newton en France au XVIII ème siècle. Blanchard, Paris

Carciofi AC, Domiciano de Souza A, Magalhães AM, Bjorkman JE, Vakili F (2008) Astrophys J Lett 676:L41

Chandrasekhar S (1933) Mon Not R Astron Soc 93:539

Che X, Monnier JD, Zhao M, Pedretti E, Thureau N, Mérand A, ten Brummelaar T, McAlister H, Ridgway ST, Turner N, Sturmann J, Sturmann L (2011) Astrophys J 732:68

Christensen-Dalsgaard J (2000) In: Breger M, Montgomery M (eds) Delta Scuti and related stars. Astronomical Society of the Pacific conference series, vol 210, p 187

Ciardi DR, van Belle GT, Akeson RL, Thompson RR, Lada EA, Howell SB (2001) Astrophys J 559:1147

Claret A (1998) Astron Astrophys Suppl Ser 131:395

Claret A (2000) Astron Astrophys 359:289

Claret A (2005) Astron Astrophys 440:647

Claret A, Hauschildt PH (2003) Astron Astrophys 412:241

Colavita MM, Wallace JK, Hines BE, Gursel Y, Malbet F, Palmer DL, Pan XP, Shao M, Yu JW, Boden AF, Dumont PJ, Gubler J, Koresko CD, Kulkarni SR, Lane BF, Mobley DW, van Belle GT (1999) Astrophys J 510:505

Collins GW II (1963) Astrophys J 138:1134

Collins GW II (1965) Astrophys J 142:265

Collins GW II, Harrington JP (1966) Astrophys J 146:152

Collins GW II, Smith RC (1985) Mon Not R Astron Soc 213:519

Collins GW II, Sonneborn GH (1977) Astrophys J Suppl Ser 34:41

Connon Smith R, Worley R (1974) Mon Not R Astron Soc 167:199 
Cotton W, Monnier J, Baron F, Hofmann K-H, Kraus S, Weigelt G, Rengaswamy S, Thiébaut E, Lawson P, Jaffe W, Hummel C, Pauls T, Schmitt H, Tuthill P, Young J (2008) In: Society of photo-optical instrumentation engineers (SPIE) conference series, vol 7013

Cox AN (ed) (2000) Allen's astrophysical quantities, 4th edn. AIP Press, New York. ISBN: 0387987460

Cranmer SR, Owocki SP (1995) Astrophys J 440:308

Cunha MS, Aerts C, Christensen-Dalsgaard J, Baglin A, Bigot L, Brown TM, Catala C, Creevey OL, Domiciano de Souza A, Eggenberger P, Garcia PJV, Grundahl F, Kervella P, Kurtz DW, Mathias P, Miglio A, Monteiro MJPFG, Perrin G, Pijpers FP, Pourbaix D, Quirrenbach A, Rousselet-Perraut K, Teixeira TC, Thévenin F, Thompson MJ (2007) Astron Astrophys Rev 14:217

Cutri RM, Skrutskie MF, van Dyk S, Beichman CA, Carpenter JM, Chester T, Cambresy L, Evans T, Fowler J, Gizis J, Howard E, Huchra J, Jarrett T, Kopan EL, Kirkpatrick JD, Light RM, Marsh KA, McCallon H, Schneider S, Stiening R, Sykes M, Weinberg M, Wheaton WA, Wheelock S, Zacarias N (2003) 2MASS All Sky Catalog of point sources

Defrère D, Absil O, Augereau J, di Folco E, Berger J, Coudé du Foresto V, Kervella P, Le Bouquin J, Lebreton J, Millan-Gabet R, Monnier JD, Olofsson J, Traub W (2011) ArXiv e-prints

Deupree RG (2011) Astrophys J 735:69

Domiciano de Souza A, Kervella P, Jankov S, Abe L, Vakili F, di Folco E, Paresce F (2003) Astron Astrophys 407:L47

Domiciano de Souza A, Kervella P, Jankov S, Vakili F, Ohishi N, Nordgren TE, Abe L (2005) Astron Astrophys 442:567

Domiciano de Souza A, Vakili F, Jankov S, Janot-Pacheco E, Abe L (2002) Astron Astrophys 393:345

Drake S (1957) Discoveries and opinions of Galileo. Doubleday Anchor, Garden City

Eddington AS (1925) The Observatory 48:73

Eddington AS (1926) The internal constitution of the stars. Dover, New York

Elvey CT (1930) Astrophys J 71:221

Engelke CW, Price SD, Kraemer KE (2010) Astron J 140:1919

Espinosa Lara F, Rieutord M (2011) Astron Astrophys 533:A43

Gerbaldi M, Faraggiana R, Balin N (2001) Astron Astrophys 379:162

Gies DR, Dieterich S, Richardson ND, Riedel AR, Team BL, McAlister HA, Bagnuolo WG Jr, Grundstrom ED, Štefl S, Rivinius T, Baade D (2008) Astrophys J Lett 682:L117

Glebocki R, Gnacinski P (2005) VizieR online data catalog, 3244, 0

Glindemann A, Algomedo J, Amestica R, Ballester P, Bauvir B, Bugueño E, Correia S, Delgado F, Delplancke F, Derie F, Duhoux P, di Folco E, Gennai A, Gilli B, Giordano P, Gitton P, Guisard S, Housen N, Huxley A, Kervella P, Kiekebusch M, Koehler B, Lévêque S, Longinotti A, Ménardi S, Morel S, Paresce F, Phan Duc T, Richichi A, Schöller M, Tarenghi M, Wallander A, Wittkowski M, Wilhelm R (2003) Astrophys Space Sci 286:35

Goodman JW (2005) In: Crisp J, Elliott B (eds) Introduction to Fourier optics

Gray DF (1980) Publ Astron Soc Pac 92:771

Gray RO (1988) J R Astron Soc Can 82:336

Gray RO (2007) In: Sterken C (ed) The future of photometric, spectrophotometric and polarimetric, standardization. Astronomical Society of the Pacific conference series, vol 364, p 305

Gray RO, Napier MG, Winkler LI (2001) Astron J 121:2148

Han C, Chang H-Y (2006) Astrophys J 645:271

Hanbury Brown R, Twiss RQ (1956) Nature 178:1046

Hanbury Brown R, Davis J, Allen LR (1974) Mon Not R Astron Soc 167:121

Haniff C (2007a) New Astron Rev 51:565

Haniff C (2007b) New Astron Rev 51:583

Harrington JP, Collins GW II (1968) Astrophys J 151:1051

Hayes DS (1967) PhD thesis, AA (University of California, Los Angeles)

Herbison-Evans D, Hanbury Brown R, Davis J, Allen LR (1971) Mon Not R Astron Soc 151:161

Hill G, Gulliver AF, Adelman SJ (2004) In: Zverko J, Ziznovsky J, Adelman SJ, Weiss WW (eds) The A-StaR puzzle. IAU symposium, vol 224, pp 35-42

Hinkley S, Monnier JD, Oppenheimer BR, Roberts LC Jr, Ireland M, Zimmerman N, Brenner D, Parry IR, Martinache F, Lai O, Soummer R, Sivaramakrishnan A, Beichman C, Hillenbrand L, Zhao M, Lloyd JP, Bernat D, Vasisht G, Crepp JR, Pueyo L, Shao M, Perrin MD, King DL, Bouchez A, Roberts JE, Dekany R, Burruss R (2011) Astrophys J 726:104

Huang W, Gies DR (2006) Astrophys J 648:591

Iorio L (2008) Astrophys Space Sci 318:51 
Ireland MJ, Monnier JD, Thureau N (2006) In: Society of Photo-Optical Instrumentation Engineers (SPIE) conference series, vol 6268

Jackson S, MacGregor KB, Skumanich A (2004) Astrophys J 606:1196

Johnson HL, Morgan WW (1953) Astrophys J 117:313

Jordahl PR (1972) PhD thesis, The University of Texas at Austin

Kanaan S, Meilland A, Stee P, Zorec J, Domiciano de Souza A, Frémat Y, Briot D (2008) Astron Astrophys 486:785

Kervella P, Domiciano de Souza A (2006) Astron Astrophys 453:1059

Kervella P, Domiciano de Souza A, Bendjoya P (2008) Astron Astrophys 484:L13

Kervella P, Domiciano de Souza A, Kanaan S, Meilland A, Spang A, Stee P (2009) Astron Astrophys 493:L53

Khalack VR (2005) Astron Astrophys 429:677

Kuhn JR, Bush RI, Emilio M, Scherrer PH (2004) Astrophys J 613:1241

Lake RJW (1975) PhD thesis, University of Sydney

Lawler SM, Beichman CA, Bryden G, Ciardi DR, Tanner AM, Su KYL, Stapelfeldt KR, Lisse CM, Harker DE (2009) Astrophys J 705:89

Lawson PR (ed) (2000) Principles of long baseline stellar interferometry

Lawson PR, Cotton WD, Hummel CA, Baron F, Young JS, Kraus S, Hofmann K-H, Weigelt GP, Ireland M, Monnier JD, Thiébaut E, Rengaswamy S, Chesneau O (2006) In: Society of Photo-Optical Instrumentation Engineers (SPIE) conference series, vol 6268

Lefebvre S, Rozelot JP, Pireaux S, Ajabshirizadeh A, Fazel Z (2005) Mem. Soc. Astron. Ital. 76:994

Lefebvre S, Rozelot JP, Kosovichev AG (2007) Adv Space Res 40:1000

Lucy LB (1967) Z Astrophys 65:89

MacGregor KB, Jackson S, Skumanich A, Metcalfe TS (2007) Astrophys J 663:560

McAlister HA, ten Brummelaar TA, Gies DR, Huang W, Bagnuolo WG Jr, Shure MA, Sturmann J, Sturmann L, Turner NH, Taylor SF, Berger DH, Baines EK, Grundstrom E, Ogden C, Ridgway ST, van Belle G (2005) Astrophys J 628:439

McLaughlin DB (1924) Astrophys J 60:22

Meynet G, Maeder A (1997) Astron Astrophys 321:465

Michelson AA, Pease FG (1921) Astrophys J 53:249

Milone EF, Leahy DA, Hobill DW (eds) (2008) Short-period binary stars: observations, analyses, and results. Astrophys. space sci. library, vol 352

Monnier JD (2003) Rep Prog Phys 66:789

Monnier JD (2007) New Astron Rev 51:604

Monnier JD, Anderson M, Baron F, Berger DH, Che X, Eckhause T, Kraus S, Pedretti E, Thureau N, Millan-Gabet R, Ten Brummelaar T, Irwin P, Zhao M (2010a) In: Society of Photo-Optical Instrumentation Engineers (SPIE) conference series, vol 7734

Monnier JD, Townsend RHD, Che X, Zhao M, Kallinger T, Matthews J, Moffat AFJ (2010b) Astrophys J 725:1192

Monnier JD, Zhao M, Pedretti E, Thureau N, Ireland M, Muirhead P, Berger J-P, Millan-Gabet R, Van Belle G, ten Brummelaar T, McAlister H, Ridgway S, Turner N, Sturmann L, Sturmann J, Berger D (2007) Science 317:342

Narayan R, Nityananda R (1986) Annu Rev Astron Astrophys 24:127

Neff JE, Simon T (2008) Astrophys J 685:478

Ohishi N, Nordgren TE, Hutter DJ (2004) Astrophys J 612:463

Orlov AA (1961) Sov Astron 4:845

Owocki SP, Cranmer SR, Blondin JM (1994) Astrophys J 424:887

Pease DO, Drake JJ, Kashyap VL (2006) Astrophys J 636:426

Perryman MAC, Lindegren L, Kovalevsky J, Hoeg E, Bastian U, Bernacca PL, Crézé M, Donati F, Grenon M, van Leeuwen F, van der Marel H, Mignard F, Murray CA, Le Poole RS, Schrijver H, Turon C, Arenou F, Froeschlé M, Petersen CS (1997) Astron Astrophys 323:L49

Peterson DM, Hummel CA, Pauls TA, Armstrong JT, Benson JA, Gilbreath GC, Hindsley RB, Hutter DJ, Johnston KJ, Mozurkewich D, Schmitt H (2006a) Astrophys J 636:1087

Peterson DM, Hummel CA, Pauls TA, Armstrong JT, Benson JA, Gilbreath GC, Hindsley RB, Hutter DJ, Johnston KJ, Mozurkewich D, Schmitt HR (2006b) Nature 440:896

Petrov RG, Malbet F, Weigelt G, Antonelli P, Beckmann U, Bresson Y, Chelli A, Dugué M, Duvert G, Gennari S, Glück L, Kern P, Lagarde S, Le Coarer E, Lisi F, Millour F, Perraut K, Puget P, Rantakyrö F, Robbe-Dubois S, Roussel A, Salinari P, Tatulli E, Zins G, Accardo M, Acke B, Agabi K, Altariba 
E, Arezki B, Aristidi E, Baffa C, Behrend J, Blöcker T, Bonhomme S, Busoni S, Cassaing F, Clausse J-M, Colin J, Connot C, Delboulbé A, Domiciano de Souza A, Driebe T, Feautrier P, Ferruzzi D, Forveille T, Fossat E, Foy R, Fraix-Burnet D, Gallardo A, Giani E, Gil C, Glentzlin A, Heiden M, Heininger M, Hernandez Utrera O, Hofmann K-H, Kamm D, Kiekebusch M, Kraus S, Le Contel D, Le Contel J-M, Lesourd T, Lopez B, Lopez M, Magnard Y, Marconi A, Mars G, Martinot-Lagarde G, Mathias P, Mège P, Monin J-L, Mouillet D, Mourard D, Nussbaum E, Ohnaka K, Pacheco J, Perrier C, Rabbia Y, Rebattu S, Reynaud F, Richichi A, Robini A, Sacchettini M, Schertl D, Schöller M, Solscheid W, Spang A, Stee P, Stefanini P, Tallon M, Tallon-Bosc I, Tasso D, Testi L, Vakili F, von der Lühe O, Valtier J-C, Vannier M, Ventura N (2007) Astron Astrophys 464:1

Porter JM, Rivinius T (2003) Publ Astron Soc Pac 115:1153

Rachford BL, Foight DR (2009) Astrophys J 698:786

Rappaport S, Podsiadlowski P, Horev I (2009) Astrophys J 698:666

Rattenbury NJ, Abe F, Bennett DP, Bond IA, Calitz JJ, Claret A, Cook KH, Furuta Y, Gal-Yam A, Glicenstein J-F, Hearnshaw JB, Hauschildt PH, Kilmartin PM, Kurata Y, Masuda K, Maoz D, Matsubara Y, Meintjes PJ, Moniez M, Muraki Y, Noda S, Ofek EO, Okajima K, Philpott L, Rhie SH, Sako T, Sullivan DJ, Sumi T, Terndrup DM, Tristram PJ, Wood J, Yanagisawa T, Yock PCM (2005) Astron Astrophys 439:645

Reiners A, Royer F (2004) Astron Astrophys 428:199

Rhee JH, Song I, Zuckerman B, McElwain M (2007) Astrophys J 660:1556

Rieke GH, Blaylock M, Decin L, Engelbracht C, Ogle P, Avrett E, Carpenter J, Cutri RM, Armus L, Gordon K, Gray RO, Hinz J, Su K, Willmer CNA (2008) Astron J 135:2245

Rieutord M (2006) In: Rieutord M, Dubrulle B (eds) EAS publications series. EAS publications series, vol 21, pp 275-295

Robrade J, Schmitt JHMM (2009) Astron Astrophys 497:511

Roche E (1837) Mém Acad Montpellier 8:235

Rossiter RA (1924) Astrophys J 60:15

Royer F (2005) Memorie. Mem Soc Astron Ital Suppl 8:124

Rozelot J-P, Lefebvre S, Desnoux V (2003) Sol Phys 217:39

Sackmann IJ (1970) Astron Astrophys 8:76

Schlesinger F (1909) Publ. Publ Allegheny Obs Univ Pittsbg 1:134

Schlesinger F (1911) Mon Not R Astron Soc 71:719

Seager S, Hui L (2002) Astrophys J 574:1004

Shajn G, Struve O (1929) Mon Not R Astron Soc 89:222

Slettebak A (1949) Astrophys J 110:498

Slettebak A (1954) Astrophys J 119:146

Slettebak A (1955) Astrophys J 121:653

Slettebak A (1982) Astrophys J Suppl Ser 50:55

Slettebak A (1985) In: Hayes DS, Pasinetti LE, Philip AGD (eds) Calibration of fundamental stellar quantities. IAU symposium, vol 111. Springer, Berlin, pp 163-183

Slettebak A, Kuzma TJ, Collins GW II (1980) Astrophys J 242:171

Smalley B, Kupka F (2003) In: Piskunov N, Weiss WW, Gray DF (eds) Modelling of stellar atmospheres. IAU symposium, vol 210, p 10P

Smalley B, Gardiner RB, Kupka F, Bessell MS (2002) Astron Astrophys 395:601

Snodgrass HB, Ulrich RK (1990) Astrophys J 351:309

Straizys V, Sudzius J, Kuriliene G (1976) Astron Astrophys 50:413

Struve O, Elvey CT (1931) Mon Not R Astron Soc 91:663

Takeda Y, Kawanomoto S, Ohishi N (2008) Astrophys J 678:446

Tassoul J-L (1978) Theory of rotating stars Princeton series in astrophysics. Princeton University Press, Princeton

Tassoul J-L (2000) In: Tassoul J-L (ed) Stellar rotation

Temple R (1986) The genius of China: 3000 years of science, discovery, and invention. Simon and Schuster, New York

ten Brummelaar TA, McAlister HA, Ridgway ST, Bagnuolo WG Jr, Turner NH, Sturmann L, Sturmann J, Berger DH, Ogden CE, Cadman R, Hartkopf WI, Hopper CH, Shure MA (2005) Astrophys J 628:453

Townes CH (1999) Astrophys J 525:C148

Trimble V, Aschwanden MJ (2003) Publ Astron Soc Pac 115:514

van Belle GT (1999) Publ Astron Soc Pac 111:1515

van Belle GT (2008) Publ Astron Soc Pac 120:617 
van Belle GT, Ciardi DR, Thompson RR, Akeson RL, Lada EA (2001) Astrophys J 559:1155

van Belle GT, Ciardi DR, ten Brummelaar T, McAlister HA, Ridgway ST, Berger DH, Goldfinger PJ, Sturmann J, Sturmann L, Turner N, Boden AF, Thompson RR, Coyne J (2006) Astrophys J 637:494 Vogel HC (1877) Astron Nachr 90:71

von Zeipel H (1924a) Mon Not R Astron Soc 84:665

von Zeipel H (1924b) Mon Not R Astron Soc 84:684

Weigelt G, Grinin VP, Groh JH, Hofmann K-H, Kraus S, Miroshnichenko AS, Schertl D, Tambovtseva LV, Benisty M, Driebe T, Lagarde S, Malbet F, Meilland A, Petrov R, Tatulli E (2011) Astron Astrophys 527:A103

Westgate C (1933a) Astrophys J 77:141

Westgate C (1933b) Astrophys J 78:46

Westgate C (1934) Astrophys J 79:357

Wilson ER (1917) Rev Pop Astron 25:88

Winn JN, Noyes RW, Holman MJ, Charbonneau D, Ohta Y, Taruya A, Suto Y, Narita N, Turner EL, Johnson JA, Marcy GW, Butler RP, Vogt SS (2005) Astrophys J 631:1215

Yi S, Demarque P, Kim Y-C, Lee Y-W, Ree CH, Lejeune T, Barnes S (2001) Astrophys J Suppl Ser 136:417

Yoon J, Peterson DM, Zagarello RJ, Armstrong JT, Pauls T (2008) Astrophys J 681:570

Yoon J, Peterson DM, Kurucz RL, Zagarello RJ (2010) Astrophys J 708:71

Zhao M, Monnier J, Pedretti E, Thureau NAM, ten Brummelaar TA, McAlister H, Ridgway ST, Turner N, Sturmann J, Sturmann L, Goldfinger P, Farrington C (2009) Astrophys J 701:209-224. doi:10.1088/0004-637X/701/1/209

Zhao M, Monnier JD, Che X, Pedretti E, Thureau N, Schaefer G, Ten Brummelaar T, Mérand A, Ridgway ST, McAlister H, Turner N, Sturmann J, Sturmann L, Goldfinger PJ, Farrington C (2011) Publ Astron Soc Pac 123:964

Zorec J, Frémat Y, Domiciano de Souza A, Delaa O, Stee P, Mourard D, Cidale L, Martayan C, Georgy C, Ekström S (2011) Astron Astrophys 526:A87 